\title{
Optimal error bounds for two-grid schemes applied to the Navier-Stokes equations
}

\author{
Javier de Frutos ${ }^{1} \quad$ Bosco García-Archilla ${ }^{2} \quad$ Julia Novo $^{3}$
}

August 19, 2018

\footnotetext{
${ }^{1}$ Departamento de Matemática Aplicada, Universidad de Valladolid. Spain. Research supported by Spanish MICINN under grant MTM2010-14919, and by JCyL grant VA001A10-1 (frutos@mac.uva.es)

${ }^{2}$ Departamento de Matemática Aplicada II, Universidad de Sevilla, Sevilla, Spain. Research supported by Spanish MICINN under grant MTM2009-07849 (bosco@esi.us.es)

${ }^{3}$ Departamento de Matemáticas, Universidad Autónoma de Madrid, Instituto de Ciencias Matemáticas CSIC-UAM-UC3M-UCM, Spain. Research supported by Spanish MICINN under grant MTM2010-14919 (julia.novo@uam.es)
} 


\begin{abstract}
We consider two-grid mixed-finite element schemes for the spatial discretization of the incompressible Navier-Stokes equations. A standard mixed-finite element method is applied over the coarse grid to approximate the nonlinear Navier-Stokes equations while a linear evolutionary problem is solved over the fine grid. The previously computed Galerkin approximation to the velocity is used to linearize the convective term. For the analysis we take into account the lack of regularity of the solutions of the Navier-Stokes equations at the initial time in the absence of nonlocal compatibility conditions of the data. Optimal error bounds are obtained.
\end{abstract}




\section{Introduction}

In this paper we study two-grid mixed finite-element (MFE) methods for the spatial discretization of the incompressible Navier-Stokes equations

$$
\begin{aligned}
u_{t}-\Delta u+(u \cdot \nabla) u+\nabla p & =f, \\
\operatorname{div}(u) & =0,
\end{aligned}
$$

in a bounded domain $\Omega \subset \mathbb{R}^{d}(d=2,3)$ with a smooth boundary subject to homogeneous Dirichlet boundary conditions $u=0$ on $\partial \Omega$. In (11), $u$ is the velocity field, $p$ the pressure, and $f$ a given force field. As in [23, 24], 25] we assume that the fluid density and viscosity have been normalized by an adequate change of scale in space and time. We approximate the solution $u$ and $p$ corresponding to a given initial condition

$$
u(\cdot, 0)=u_{0} .
$$

Two-grid methods are a well established technique for nonlinear steady problems, see [34], 35]. The main idea in a two-level method involves the discretization of the equations over two meshes of different size. A nonlinear system over the coarse mesh is solved in the first step of the method. In a second step, a linearized equation based on the approximation over the coarse mesh is solved on the fine mesh. In [28, [29] several two-level methods are considered to approximate the steady Navier-Stokes equations. In these papers, depending on the algorithm, the second step is based on the solution of a discrete Stokes problem, a linear Oseen problem or one step of the Newton method over the fine mesh with the coarse mesh approximation as initial guess.

Several two-level or two-grid schemes have also been considered in the literature to approximate the evolutionary nonlinear Navier-Stokes equations (11)-(2). Again, two approximations to the velocity (and correspondingly two approximations to the pressure), are computed. One of them is defined by a discretization of the nonlinear equations over a coarse mesh, $u_{H}$, and another one, $\tilde{u}_{h}$, is defined by an appropriate linearization over a fine mesh. Different classes of algorithms can be seen as two level methods. In particular, although they were originally developed from different ideas, the so called nonlinear Galerkin methods, postprocessed and dynamical postprocessed methods, fall into this category.

Postprocessed Galerkin methods were first introduced for Fourier spectral methods in [18], [19] (see also [31]) and later extended to finite element methods in [7], 6], 14], [15]. In all these works the main idea is the following: 
one first compute the standard Galerkin approximation to the velocity and pressure over a coarse mesh $\left(u_{H}, p_{H}\right)$ of size $H$ and then compute the postprocessed approximation in a finer mesh at selected times in which one wants to obtain an improved approximation. More precisely, the postprocessed approximation $\left(\tilde{u}_{h}, \tilde{p}_{h}\right)$ computed at a given time $t^{*}$ is an approximation in a mesh of size $h \ll H$ to the following (steady) Stokes problem:

$$
\begin{aligned}
& \left.\begin{array}{r}
-\Delta \tilde{u}+\nabla \tilde{p}=f-\frac{d}{d t} u_{H}\left(t^{*}\right)-\left(u_{H}\left(t^{*}\right) \cdot \nabla\right) u_{H}\left(t^{*}\right) \\
\operatorname{div}(\tilde{u})=0
\end{array}\right\} \quad \text { in } \Omega, \\
& \tilde{u}=0, \quad \text { on } \partial \Omega \text {. }
\end{aligned}
$$

Here, $u_{H}(t), t \in(0, T]$, is the standard MFE approximation computed in the coarse mesh in a time interval $(0, T]$ and $t^{*} \in(0, T]$. Note that the computation of $\left(u_{H}(t), p_{H}(t)\right), t \in(0, T]$, is completely independent of the computation of $\left(\tilde{u}_{h}\left(t^{*}\right), \tilde{p}_{h}\left(t^{*}\right)\right)$ in the fine mesh. The postprocessed approximation improves the rate of convergence of the standard Galerkin approximation over the coarse mesh in the following sense. If the rate of convergence of the Galerkin approximation to the velocity in the $L^{2}(\Omega)^{d}(j=0)$ or $H^{1}(\Omega)^{d}$ $(j=1)$ norm is $O\left(H^{r-j}\right)$ then the rate of convergence of the postprocessed approximation to the velocity is $O\left(H^{r+1-j}|\log (H)|\right)+O\left(h^{r-j}\right)$. Analogous results are obtained for the pressure. For first order mixed finite element methods the improvement in the rate of convergence of the velocity is only achieved in the $H^{1}(\Omega)^{d}$ norm, [6]. Then, if one wants to achieve the optimal accuracy of the fine level in the $H^{1}(\Omega)^{d}$ norm, one can first compute the Galerkin approximation on a coarse mesh of size $H=h^{(r-1) / r}$ and then compute the postprocessed approximation over the fine mesh of size $h$ at the desired time levels. For example, one should take $H=h^{1 / 2}$ and $H=h^{2 / 3}$ for linear and quadratic mixed finite elements, respectively. It can be expected that the computational cost of the postprocessed approximation is smaller than that of the Galerkin approximation on the same fine mesh, since in the former method the time evolution is done on the coarse mesh, and only at selected time levels are computations done on the fine mesh. This has been confirmed by the numerical experiments in [7] (see also [16] and [18])

In [31] a related algorithm, the so-called dynamical postprocessing, is introduced for the Fourier case. In this algorithm, the standard Galerkin approximation, $\left(u_{H}, p_{H}\right)$, is computed over a coarse mesh in the first level, as before. For the second level an approximation to a linear evolutionary problem, instead of the steady problem (3), is computed. More precisely, the dynamical postprocessing involves the approximation, at each time step, 
over a mesh of size $h \ll H$ of the problem:

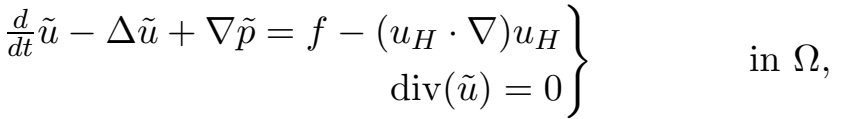

$$
\begin{aligned}
& \tilde{u}=0, \quad \text { on } \partial \Omega .
\end{aligned}
$$

Note that in the dynamical postprocessing, the computation of $\left(u_{H}(t), p_{H}(t)\right)$ and $\left(\tilde{u}_{h}(t), \tilde{p}_{h}(t)\right), t \in[0, T]$, is coupled. The rate of convergence of the dynamical postprocessing scheme is proved in [31] to be the same as the rate of convergence of the standard postprocessing. In the case of highly oscillatory solutions the dynamical algorithm is shown to be more efficient than the standard postprocessing in some one dimensional examples. The dynamical postprocessing method is also considered in [33, named now as two-level method, in the case of mixed finite elements. In [33], the author treats the fully discrete case integrating in time with the backward Euler method. A similar two-level scheme is also considered and analyzed in [21] where the author uses first order mixed finite elements in space, Crank-Nicolson extrapolation for the time integration over the coarse mesh and the backward Euler method for the time integration over the fine mesh.

The so-called nonlinear Galerkin methods are also two-level methods that have been used to compute approximations to (11)-(2). They were first introduced for Fourier spectral methods [12], 32, and later extended to mixed finite element methods in [5]. In this work the authors obtain the rate of convergence of the nonlinear Galerkin method in the case of first order elements. The rate of convergence is the same one of the postprocessed method. The main difference between the nonlinear Galerkin methods and the postprocessed or two-grid methods is that in the former the approximation on the coarse mesh takes into account the influence of the fine mesh, whereas in the latter it is just the standard Galerkin method (i.e., computed independently of the fine mesh).

In this paper we analyze two two-grid algorithms in the context of spatial mixed finite element discretizations to approximate the solutions of (11)-(2). The two algorithms we consider are very similar to the dynamical postprocessing method. The difference is the treatment of the nonlinearity in the second level. In the dynamical postprocessing method the nonlinear convective term of the fine level is approximated by the data $\left(u_{H} \cdot \nabla\right) u_{H}$ (see the right-hand-side of (4)). In the two algorithms we consider in the present paper, the approximation to the velocity of the coarse mesh $u_{H}$ is used to linearize the nonlinear convective term of the fine level. In the first algorithm, the linearized convective term of the fine level is $\left(u_{H} \cdot \nabla\right) \tilde{u}_{h}$. In the 
second algorithm $u_{H}$ is regarded as an initial guess to perform one Newton step in the fine level. For the spatial discretization we consider mixed finite elements of first, second and third order. More precisely, we consider the mini-element and the quadratic and cubic Hood-Taylor elements. The analysis for other mixed finite elements of the same order is similar. As in 24, [14] due to the lack of regularity at $t=0$ of the solution of (11)-(2) no better than $O\left(H^{5}\right)$ error bounds can be expected. For this reason we do not analyze higher than cubic finite element discretizations. For the temporal discretization we use the backward Euler method or the two-step backward differentiation formula. The analysis of the fully discrete methods is similar to the one appeared in [15] and it is only briefly indicated in this paper.

This is not the first time these two algorithms have been considered. The first algorithm was introduced in [20, where the authors analyze the semidiscrete in space case for first order finite elements. In [2] the authors extend this analysis to the fully discrete case and in [1] the second order Hood-Taylor finite element is used for the spatial discretization and the two-step backward differentiation formula for the time integration. In [27] the second algorithm is analyzed for the Fourier spectral case while in [30] the analysis is extended to the case of first order mixed finite elements considering the fully discrete case coupled with the Crank-Nicolson scheme for the time integration. As opposed to the above mentioned works on the same methods, in the present paper we take into account the lack of regularity suffered by the solutions of the Navier-Stokes equations at the initial time. Then, for the analysis in the present paper we do not assume more than second-order spatial derivatives bounded in $L^{2}$ up to initial time $t=0$, since demanding further regularity requires the data to satisfy nonlocal compatibility conditions unlikely to be fulfilled in practical situations [23, 24]. This is the first time these methods are analyzed under realistic regularity assumptions. Also, this is the first time the cubic case is considered and the first time the quadratic case is considered for the second method.

There are some other improvements with respect to previous works. In 11 the authors get an error bound of order $O\left(H^{3}+h^{2}+(\Delta t)^{2}\right)$ for the fine approximation to the velocity $\tilde{u}_{h}$ in the $H^{1}(\Omega)^{d}$ norm whenever the following inequality is satisfied $\alpha_{1} H^{3} \leq(\Delta t)^{2} \leq \alpha_{2} H^{3}, \alpha_{1}$ and $\alpha_{2}$ being constants independent of $H$ and $\Delta t$. With the technique of this paper an error bound of order $O\left(|\log (h)||\log (H)| H^{4}+h^{2}+(\Delta t)^{2}\right)$ for the same fully discrete method in the $H^{1}(\Omega)^{d}$ norm can be obtained for $H$ and $\Delta t$ independently chosen. With the new error bound obtained in this paper one can achieve the rate of convergence of the fine mesh in the $H^{1}(\Omega)^{d}$ norm by taking $H=h^{1 / 2}$ instead of $H=h^{2 / 3}$. This fact improves the efficiency of the 
method compared with the (same order) standard Galerkin method over the fine mesh. Also, the authors of [1] remark that they have observed the same rate of convergence for the two-grid method with $H=h^{1 / 2}$ and $H=h^{2 / 3}$ in the numerical tests they have carried out, which supports the improved rate of convergence we obtain in this paper. We want to remark that in all the numerical experiments of [27, 30] and [1] the two-grid algorithms improve the efficiency of the standard Galerkin method in the sense that a given error can be achieved with less computational cost with the new algorithms than with the standard Galerkin method. In [27] a comparison in the Fourier case between the standard postprocessing, the dynamical postprocessing and the second two-grid algorithm is also included. Although the computational cost of the two-grid approximation over the fine mesh is bigger than that of the postprocessed approximations, the two-grid algorithm produces smaller errors in the case of moderate to high Reynolds numbers. Finally, comparing the two algorithms we analyze in this paper we remark that with the second algorithm better error bounds are obtained in terms of $H$. Although this fact could make the choice of the second algorithm preferable for computations, it turns out in practice to be rather inefficient to solve the linear systems accurately. For this reason, some authors suggest solving instead an Oseen problem leading then to the first algorithm, see [28].

The rest of the paper is as follows. In Section 2 we introduce some preliminaries and notations. In Section 3 we carry out the error analysis of the first two-grid algorithm in the semi-discrete in space case. In Section 4 we consider the analysis of the second two-grid algorithm in the semidiscrete in space case. Finally, in Section 5 we consider the fully discrete case integrating in time with the backward Euler method or the two-step backward differentiation formula.

\section{Preliminaries and notations}

We will assume that $\Omega$ is a bounded domain in $\mathbb{R}^{d}, d=2,3$, not necessarily convex and smooth enough. When dealing with linear elements $(r=2$ below) $\Omega$ may also be a convex polygonal or polyhedral domain. We will consider the Hilbert spaces

$$
\begin{aligned}
& H=\left\{u \in\left(L^{2}(\Omega)\right)^{d} \mid \operatorname{div}(u)=0, u \cdot n_{\left.\right|_{\partial \Omega}}=0\right\}, \\
& V=\left\{u \in\left(H_{0}^{1}(\Omega)\right)^{d} \mid \operatorname{div}(u)=0\right\},
\end{aligned}
$$

endowed with the inner product of $L^{2}(\Omega)^{d}$ and $H_{0}^{1}(\Omega)^{d}$, respectively. For $l \geq$ 0 integer and $1 \leq q \leq \infty$, we consider the standard Sobolev spaces $W^{l, q}(\Omega)^{d}$ 
of functions with derivatives up to order $l$ in $L^{q}(\Omega)$, and $H^{l}(\Omega)^{d}=W^{l, 2}(\Omega)^{d}$. We will denote by $\|\cdot\|_{l}$ the norm in $H^{l}(\Omega)^{d}$, and $\|\cdot\|_{-l}$ will represent the norm of its dual space. We consider also the quotient spaces $H^{l}(\Omega) / \mathbb{R}$ with norm $\|p\|_{H^{l} / \mathbb{R}}=\inf \left\{\|p+c\|_{l} \mid c \in \mathbb{R}\right\}$.

Let us recall the following Sobolev's imbedding inequalities [4]: For $q \in$ $[1, \infty)$, there exists a constant $C=C(\Omega, q)$ such that

$$
\|v\|_{L^{q^{\prime}}(\Omega)^{d}} \leq C\|v\|_{W^{s, q}(\Omega)^{d}}, \quad \frac{1}{q^{\prime}} \geq \frac{1}{q}-\frac{s}{d}>0, \quad q<\infty, \quad v \in W^{s, q}(\Omega)^{d} .
$$

For $q^{\prime}=\infty$, (5) holds with $\frac{1}{q}<\frac{s}{d}$.

Let $\Pi: L^{2}(\Omega)^{d} \longrightarrow H$ be the $L^{2}(\Omega)^{d}$ projection onto $H$. We denote by $A$ the Stokes operator on $\Omega$ :

$$
A: \mathcal{D}(A) \subset H \longrightarrow H, \quad A=-\Pi \Delta, \quad \mathcal{D}(A)=H^{2}(\Omega)^{d} \cap V .
$$

Applying Leray's projector to (11), the equations can be written in the form

$$
u_{t}+A u+B(u, u)=\Pi f \quad \text { in } \varnothing,
$$

where $B(u, v)=\Pi(u \cdot \nabla) v$ for $u, v$ in $H_{0}^{1}(\Omega)^{d}$.

We shall use the trilinear form $b(\cdot, \cdot, \cdot)$ defined by

$$
b(u, v, w)=(F(u, v), w) \quad \forall u, v, w \in H_{0}^{1}(\Omega)^{d},
$$

where

$$
F(u, v)=(u \cdot \nabla) v+\frac{1}{2}(\nabla \cdot u) v \quad \forall u, v \in H_{0}^{1}(\Omega)^{d} .
$$

It is straightforward to verify that $b$ enjoys the skew-symmetry property

$$
b(u, v, w)=-b(u, w, v) \quad \forall u, v, w \in H_{0}^{1}(\Omega)^{d} .
$$

Let us observe that $B(u, v)=\Pi F(u, v)$ for $u \in V, v \in H_{0}^{1}(\Omega)^{d}$.

We shall assume that

$$
\|u(t)\|_{1} \leq M_{1}, \quad\|u(t)\|_{2} \leq M_{2}, \quad 0 \leq t \leq T
$$

and, for $k \geq 2$ integer,

$$
\sup _{0 \leq t \leq T}\left\|\partial_{t}^{\lfloor k / 2\rfloor} f\right\|_{k-1-2\lfloor k / 2\rfloor}+\sum_{j=0}^{\lfloor(k-2) / 2\rfloor} \sup _{0 \leq t \leq T}\left\|\partial_{t}^{j} f\right\|_{k-2 j-2}<+\infty,
$$


so that, according to Theorems 2.4 and 2.5 in [23], there exist positive constants $M_{k}$ and $K_{k}$ such that for $k \geq 2$

$$
\|u(t)\|_{k}+\left\|u_{t}(t)\right\|_{k-2}+\|p(t)\|_{H^{k-1} / \mathbb{R}} \leq M_{k} \tau(t)^{1-k / 2}
$$

and for $k \geq 3$

$$
\int_{0}^{t} \sigma_{k-3}(s)\left(\|u(s)\|_{k}^{2}+\left\|u_{s}(s)\right\|_{k-2}^{2}+\|p(s)\|_{H^{k-1} / \mathbb{R}}^{2}+\left\|p_{s}(s)\right\|_{H^{k-3} / \mathbb{R}}^{2}\right) d s \leq K_{k}^{2},
$$

where $\tau(t)=\min (t, 1)$ and $\sigma_{n}=e^{-\alpha(t-s)} \tau^{n}(s)$ for some $\alpha>0$. Observe that, for $t \leq T<\infty$, we can take $\tau(t)=t$ and $\sigma_{n}(s)=s^{n}$. For simplicity, we will take these values of $\tau$ and $\sigma_{n}$. We note that no further than $k \leq 6$ will be needed in the present paper.

Let $\mathcal{T}_{h}=\left(\tau_{i}^{h}, \phi_{i}^{h}\right)_{i \in I_{h}}, h>0$, be a family of partitions of suitable domains $\Omega_{h}$, where $h$ is the maximum diameter of the elements $\tau_{i}^{h} \in \mathcal{T}_{h}$ and $\phi_{i}^{h}$ are the mappings of the reference simplex $\tau_{0}$ onto $\tau_{i}^{h}$. We restrict ourselves to quasi-uniform and regular meshes $\mathcal{T}_{h}$.

Let $r \geq 2$, we consider the finite-element spaces

$$
\begin{aligned}
& S_{h, r}=\left\{\chi_{h} \in \mathcal{C}\left(\bar{\Omega}_{h}\right)\left|\chi_{h}\right|_{\tau_{i}^{h}} \circ \phi_{i}^{h} \in P^{r-1}\left(\tau_{0}\right)\right\}, \\
& S_{h, r}^{0}=\left\{\chi_{h} \in \mathcal{C}\left(\bar{\Omega}_{h}\right)\left|\chi_{h}\right|_{\tau_{i}^{h}} \circ \phi_{i}^{h} \in P^{r-1}\left(\tau_{0}\right), \chi_{h}(x)=0 \forall x \in \partial \Omega_{h}\right\},
\end{aligned}
$$

where $P^{r-1}\left(\tau_{0}\right)$ denotes the space of polynomials of degree at most $r-1$ on $\tau_{0}$. Since we are assuming that the meshes are quasi-uniform, the following inverse inequality holds for each $v_{h} \in\left(S_{h, r}^{0}\right)^{d}$ (see, e.g., [10, Theorem 3.2.6])

$$
\left\|v_{h}\right\|_{W^{m, q}(\tau)^{d}} \leq C h^{l-m-d\left(\frac{1}{q^{\prime}}-\frac{1}{q}\right)}\left\|v_{h}\right\|_{W^{l, q^{\prime}}(\tau)^{d}},
$$

where $0 \leq l \leq m \leq 1,1 \leq q^{\prime} \leq q \leq \infty$, and $\tau$ is an element in the partition $\mathcal{T}_{h}$.

We shall denote by $\left(X_{h, r}, Q_{h, r-1}\right)$ the so-called Hood-Taylor element [8, 26], when $r \geq 3$, where

$$
X_{h, r}=\left(S_{h, r}^{0}\right)^{d}, \quad Q_{h, r-1}=S_{h, r-1} \cap L^{2}\left(\varnothing_{h}\right) / \mathbb{R}, \quad r \geq 3,
$$

and the so-called mini-element [9] when $r=2$, where $Q_{h, 1}=S_{h, 2} \cap L^{2}\left(\varnothing_{h}\right) / \mathbb{R}$, and $X_{h, 2}=\left(S_{h, 2}^{0}\right)^{d} \oplus \mathbb{B}_{h}$. Here, $\mathbb{B}_{h}$ is spanned by the bubble functions $b_{\tau}$, $\tau \in \mathcal{T}_{h}$, defined by $b_{\tau}(x)=(d+1)^{d+1} \lambda_{1}(x) \cdots \lambda_{d+1}(x)$, if $x \in \tau$ and 0 elsewhere, where $\lambda_{1}(x), \ldots, \lambda_{d+1}(x)$ denote the barycentric coordinates of $x$. 
For these mixed elements a uniform inf-sup condition is satisfied (see [8]); that is, there exists a constant $\beta>0$ independent of the mesh grid size $h$ such that

$$
\inf _{q_{h} \in Q_{h, r-1}} \sup _{v_{h} \in X_{h, r}} \frac{\left(q_{h}, \nabla \cdot v_{h}\right)}{\left\|v_{h}\right\|_{1}\left\|q_{h}\right\|_{L^{2} / \mathbb{R}}} \geq \beta .
$$

The approximate velocity belongs to the discretely divergence-free space

$$
V_{h, r}=X_{h, r} \cap\left\{\chi_{h} \in H_{0}^{1}\left(\Omega_{h}\right) \mid\left(q_{h}, \nabla \cdot \chi_{h}\right)=0 \quad \forall q_{h} \in Q_{h, r-1}\right\} .
$$

We observe that, for the Hood-Taylor element, $V_{h, r}$ is not a subspace of $V$. Let $\Pi_{h}: L^{2}(\varnothing)^{d} \longrightarrow V_{h, r}$ be the discrete Leray's projection defined by

$$
\left(\Pi_{h} u, \chi_{h}\right)=\left(u, \chi_{h}\right) \quad \forall \chi_{h} \in V_{h, r} .
$$

We will use the following well known bounds

$$
\left\|\left(I-\Pi_{h}\right) u\right\|_{j} \leq C h^{l-j}\|u\|_{l}, \quad 1 \leq l \leq r, \quad j=0,1 .
$$

Assuming that $\Omega$ is has a smooth enough boundary, we also have

$$
\left\|A^{-m / 2} \Pi\left(I-\Pi_{h}\right) u\right\|_{0} \leq C h^{l+\min (m, r-2)}\|u\|_{l}, \quad 1 \leq l \leq r, \quad m=1,2 .
$$

Since $\left(A_{h}^{-1 / 2} \Pi_{h} f, v_{h}\right)=\left(f, A_{h}^{-1 / 2} v_{h}\right)$, for all $v_{h} \in V_{h, r}$, it follows that

$$
\left\|A_{h}^{-1 / 2} \Pi_{h} f\right\|_{0} \leq C\|f\|_{-1} \text {. }
$$

Moreover it holds for $f \in L^{2}(\Omega)^{d}$, see [14]:

$$
\left\|A_{h}^{-s / 2} \Pi_{h} f\right\|_{0} \leq C h^{s}\|f\|_{0}+\left\|A^{-s / 2} \Pi f\right\|_{0} \quad s=1,2 .
$$

Let $\mathcal{A}$ denote either $\mathcal{A}=A$ or $\mathcal{A}=A_{h}$. Notice that both are positive selfadjoint operators with compact resolvent in $H$ and $V_{h}$, respectively. Let us consider then for $\alpha \in \mathbb{R}$ and $t>0$ the operators $\mathcal{A}^{\alpha}$ and $e^{-t \mathcal{A}}$, which are defined by means of the spectral properties of $\mathcal{A}$ (see, e.g., [11, p. 33], [17]). An easy calculation shows that

$$
\left\|\mathcal{A}^{\alpha} e^{-t \mathcal{A}}\right\|_{0} \leq\left(\alpha e^{-1}\right)^{\alpha} t^{-\alpha}, \quad \alpha \geq 0, t>0,
$$

where, here and in what follows, $\|\cdot\|_{0}$ when applied to an operator denotes the associated operator norm. Also, using the change of variables $\tau=s / t$, it is easy to show that

$$
\int_{0}^{t} s^{-1 / 2}\left\|A_{h}^{1 / 2} e^{-(t-s) A_{h}}\right\|_{0} d s \leq \frac{1}{\sqrt{2 e}} B\left(\frac{1}{2}, \frac{1}{2}\right),
$$

where $B$ is the Beta function (see, e.g., [3]). 


\section{Semi-discretization in space. The first two-grid algorithm.}

In this section we carry out the error analysis of the first two-grid algorithm for the Hood-Taylor mixed finite element with $r=3$ or 4 . At the end of the section we include the results that can be obtained for the mini-element with a similar but simpler analysis than the one showed along the section.

The first algorithm we consider is the following. Let us choose $h<H$ so that $V_{H, r} \subset V_{h, r}$. Then, in the first level we compute the standard mixed finite-element approximation to (11)-(2). That is, given $u_{H}(0)=\Pi_{H}\left(u_{0}\right)$, we compute $u_{H}(t) \in X_{H, r}$ and $p_{H}(t) \in Q_{H, r-1}, t \in(0, T]$, satisfying, for all $\phi_{H} \in X_{H, r}$ and $\psi_{H} \in Q_{H, r-1}$

$$
\begin{aligned}
\left(\dot{u}_{H}, \phi_{H}\right)+\left(\nabla u_{H}, \nabla \phi_{H}\right)+b\left(u_{H}, u_{H}, \phi_{H}\right)+\left(\nabla p_{H}, \phi_{H}\right) & =\left(f, \phi_{H}\right) \\
\left(\nabla \cdot u_{H}, \psi_{H}\right) & =0 .
\end{aligned}
$$

In the second level we solve a linearized problem on a finer grid and given $\tilde{u}_{h}(0)=\Pi_{h} u_{0}$ we compute $\tilde{u}_{h}(t) \in X_{h, r}$ and $\tilde{p}_{h}(t) \in Q_{h, r-1}, t \in(0, T]$, satisfying, for all $\phi_{h} \in X_{h, r}$ and $\psi_{h} \in Q_{h, r-1}$

$$
\begin{aligned}
\left(\dot{\tilde{u}}_{h}, \phi_{h}\right)+\left(\nabla \tilde{u}_{h}, \nabla \phi_{h}\right)+\left(u_{H} \cdot \nabla \tilde{u}_{h}, \phi_{h}\right)+\left(\nabla \tilde{p}_{h}, \phi_{h}\right) & =\left(f, \phi_{h}\right) \\
\left(\nabla \cdot \tilde{u}_{h}, \psi_{h}\right) & =0 .
\end{aligned}
$$

To obtain the error bounds for $\left(\tilde{u}_{h}, \tilde{p}_{h}\right)$ we will follow the error analysis of [14] and introduce an auxiliary approximation (see [14, Section 4.1]). For a $u$ and $p$ solution of (11)-(2) let us consider the approximations $v_{h}:[0, T] \longrightarrow X_{h, r}$ and $g_{h}:[0, T] \longrightarrow Q_{h, r-1}$, respectively, solutions of

$$
\begin{aligned}
\left(\dot{v}_{h}, \phi_{h}\right)+\left(\nabla v_{h}, \nabla \phi_{h}\right)+\left(\nabla g_{h}, \phi_{h}\right) & =\left(f, \phi_{h}\right)-b\left(u, u, \phi_{h}\right) \\
\left(\nabla \cdot v_{h}, \psi_{h}\right) & =0,
\end{aligned}
$$

for all $\phi_{h} \in X_{h, r}$ and $\psi_{h} \in Q_{h, r-1}$, with initial condition $v_{h}(0)=\Pi_{h} u_{0}$. We will also use the following notation:

$$
z_{h}=\Pi_{h} u-v_{h}
$$

Next, we state some lemmas that are needed in the proof of the main theorems. The first one summarizes previous results. 
Lemma 1 Let $(u, p)$ be the solution of (11)-(2). There exists a positive constant $C$ such that the discrete velocity $v_{h}$ defined by (21)-(22) and the HoodTaylor element approximation to $u, u_{H}$, satisfy the following bounds for $j=0,1$, and $t \in(0, T]$ :

$$
\begin{aligned}
\left\|v_{H}(t)-u_{H}(t)\right\|_{j} & \leq \frac{C}{t^{(r-2) / 2}}|\log (H)| H^{r+1-j}, \quad 3 \leq r \leq 4, \\
\left\|u_{H}(t)-u(t)\right\|_{j} & \leq \frac{C}{t^{(r-2) / 2}} H^{r-j}, \quad 2 \leq r \leq 5, \\
\int_{0}^{t}\left\|u_{H}(s)-u(s)\right\|_{j}^{2} d s & \leq C H^{2(3-j)}, \quad 3 \leq r \leq 4 .
\end{aligned}
$$

Proof The bound (24) is proved in Theorems 4.7 and 4.15 in [14. The case $j=0$ in (26) is proved in [23, Theorem 3.1] and [24, Theorem 3.1]. The case $j=1$ follows from the case $j=0$ by applying (9) and (11), see also Corollaries 4.8 and 4.16 in [14]. Finally, (26) is proved in Lemmas 5.1 and 5.2 in [24].

For the convenience of the reader, we will reproduce here the following two Lemmas, the first one from [7] and the second one from [14].

Lemma 2 For any $f \in C\left([0, T] ; L^{2}(\varnothing)^{d}\right)$, the following estimate holds for all $t \in[0, T]:$

$$
\int_{0}^{t}\left\|A_{h} e^{-(t-s) A_{h}} \Pi_{h} f(s)\right\|_{0} d s \leq C|\log (h)| \max _{0 \leq t \leq T}\|f(t)\|_{0} .
$$

Lemma 3 Let $(u, p)$ be the solution of (11)-(2). Then, there exists a positive constant $C$ such that the error $z_{h}=\Pi_{h} u-v_{h}$ in (23) satisfies the following bound:

$$
\left\|A_{h}^{(-1+j) / 2} z_{h}\right\|_{0} \leq \frac{C}{t^{(r-2) / 2}} h^{r+1-j}, \quad j=0,1,2, \quad r \geq 3 .
$$

Lemma 4 For each $\alpha>0$ there exist positive constants $K>0$ and $h_{0}$ depending on $\alpha$ and $M_{2}$ such that, for $h<H<h_{0}, h_{1}=h_{2}=h$ or $\left\{h_{1}, h_{2}\right\}=\{h, H\}$, and every $w_{h_{1}}^{1}(\cdot) \in V_{h_{1}, r}$ and,$w_{h_{2}}^{2}(\cdot) \in V_{h_{2}, r}$ satisfying the threshold condition

$$
\left\|u(t)-w_{h_{1}}^{1}(t)\right\|_{j} \leq \alpha h_{1}^{2-j}, \quad\left\|u(t)-w_{h_{2}}^{2}(t)\right\|_{j} \leq \alpha h_{2}^{2-j}, \quad j=0,1, t \in[0, T],
$$

for $w_{h}(t) \in H_{0}^{1}(\Omega), t \in[0, T]$, satisfying

$$
\left\|w_{h}(t)\right\|_{j} \leq 2 \alpha \max \left(h_{1}, h_{2}\right)^{2-j},
$$


the following bounds hold:

$$
\begin{aligned}
\left\|\mathcal{F}\left(w_{h}, w_{h_{2}}^{2}\right)\right\|_{0}+\left\|\mathcal{F}\left(w_{h_{1}}^{1}, w_{h}\right)\right\|_{0} & \leq K\left\|w_{h}\right\|_{1}, \\
\left\|\mathcal{F}\left(w_{h}, w_{h_{2}}^{2}\right)\right\|_{-1}+\left\|\mathcal{F}\left(w_{h_{1}}^{1}, w_{h}\right)\right\|_{-1} & \leq K\left\|w_{h}\right\|_{0}, \\
\left\|\mathcal{B}_{h}\left(w_{h}, w_{h_{2}}^{2}\right)\right\|_{0}+\left\|\mathcal{B}_{h}\left(w_{h_{1}}^{1}, w_{h}\right)\right\|_{0} & \leq K\left\|w_{h}\right\|_{1}, \\
\left\|A_{h}^{-1 / 2}\left(\mathcal{B}_{h}\left(w_{h}, w_{h_{2}}^{2}\right)\right)\right\|_{0}+\left\|A_{h}^{-1 / 2}\left(\mathcal{B}_{h}\left(w_{h_{1}}^{1}, w_{h}\right)\right)\right\|_{0} & \leq K\left\|w_{h}\right\|_{0},
\end{aligned}
$$

where $\mathcal{F}(u, v)$ can be either $(u \cdot \nabla) v+\frac{1}{2}(\nabla \cdot u) v$ or $(u \cdot \nabla) v$, and $\mathcal{B}_{h}=\Pi_{h} \mathcal{F}$.

Proof The proof of the present lemma can be found in that of Lemma 4.4 in [14] for $\mathcal{F}(u, v)=(u \cdot \nabla) v+\frac{1}{2}(\nabla \cdot u) v$ and $w_{h} \in V_{h, r}$. With obvious changes, the proof is also valid when $\mathcal{F}(u, v)=(u \cdot \nabla) v$, as well as when $w_{h} \notin V_{h, r}$.

Remark 1 We will apply the above inequalities for $w_{h}=w_{h_{1}}^{1}-w_{h_{2}}^{2}$, $w_{h}=w_{h_{1}}^{1}-u$ and $w_{h}=w_{h_{2}}^{2}-u$. Let us also remark that the Lemma 4 also holds if either $w_{h_{1}}^{1}$ or $w_{h_{2}}^{2}$ is replaced by $u$. In what follows we will apply Lemma 4 to $u_{h}$ and $v_{h}$ both satisfying the threshold condition (28) for an appropriate value of $\alpha$ (see [14, Remark 4.1]).

Lemma 5 For $v \in\left(H^{2}(\Omega)\right)^{d} \cap V$ there exists a positive constant $K=$ $K\left(\|v\|_{2}\right)$ such that $w \in H_{0}^{1}(\Omega)^{d}$ the following bound holds for $e=v-w$ :

$$
\left\|A^{-1} \Pi[\mathcal{F}(v, e)+\mathcal{F}(e, v)]\right\|_{0} \leq K\|v-w\|_{-1}
$$

where $\mathcal{F}(u, v)$ can be either $(u \cdot \nabla) v+\frac{1}{2}(\nabla \cdot u) v$ or $(u \cdot \nabla) v$.

Proof The proof of this result when $\mathcal{F}(u, v)=(u \cdot \nabla) v+\frac{1}{2}(\nabla \cdot u) v$ can be found as part of the proof of [7, Lemma 3.4]. With obvious changes, the proof is also valid when $\mathcal{F}(u, v)=(u \cdot \nabla) v$.

Let us observe that the approximation over the finer grid $\tilde{u}_{h}$ and the recently defined $v_{h}$ satisfy

$$
\begin{gathered}
\dot{\tilde{u}}_{h}+A_{h} \tilde{u}_{h}+\Pi_{h}\left(u_{H} \cdot \nabla \tilde{u}_{h}\right)=\Pi_{h} f, \quad u_{h}(0)=\Pi_{h} u_{0}, \\
\dot{v}_{h}+A_{h} v_{h}+\Pi_{h}(u \cdot \nabla u)=\Pi_{h} f, \quad v_{h}(0)=\Pi_{h} u_{0},
\end{gathered}
$$

respectively. Then $e_{h}=v_{h}-\tilde{u}_{h}$ satisfies

$$
\dot{e}_{h}+A_{h} e_{h}+\Pi_{h}\left(u_{H} \cdot \nabla e_{h}\right)=\Pi_{h} \rho_{h, H}, \quad e_{h}(0)=0,
$$


where

$$
\rho_{h, H}=u_{H} \cdot \nabla v_{h}-u \cdot \nabla u .
$$

In the proof of Theorem 1 below we will use the following lemmas.

Lemma 6 Let $(u, p)$ be the solution of (11)-(21). There exists a positive constant $C$ such that the following inequality holds for $r=3,4$ :

$$
\left\|A_{h}^{-1} \Pi_{h} \rho_{h, H}\right\|_{0} \leq \frac{C}{t^{(r-2) / 2}}|\log (H)| H^{r+1}, \quad t \in(0, T] .
$$

Proof Let us write $\rho_{h, H}=\rho_{h, H}^{1}+\rho_{h, H}^{2}$, where

$$
\rho_{h, H}^{1}=\left(\left(u_{H}-u\right) \cdot \nabla v_{h}\right), \quad \rho_{h, H}^{2}=\left(u \cdot \nabla\left(v_{h}-u\right)\right) .
$$

By applying (14) we have

$$
\left\|A_{h}^{-1} \Pi_{h} \rho_{h, H}^{j}\right\|_{0} \leq C h^{2}\left\|\rho_{h, H}^{j}\right\|_{0}+\left\|A^{-1} \Pi \rho_{h, H}^{j}\right\|_{0}, \quad j=1,2 .
$$

To bound $\left\|\rho_{h, H}^{1}\right\|_{0}$ let us recall Remark 1 and apply (29) to get

$$
\left\|\rho_{h, H}^{1}\right\|_{0} \leq C\left\|u_{H}-u\right\|_{1} \leq C \frac{H^{r-1}}{t^{(r-2) / 2}}
$$

where we have applied (25) from Lemma 1 in the last inequality. Applying (29) we also get

$$
\left\|\rho_{h, H}^{2}\right\|_{0} \leq C\left\|v_{h}-u\right\|_{1} \leq C \frac{h^{r-1}}{t^{(r-2) / 2}},
$$

where in the last inequality we have applied standard bounds for $\Pi_{h}$ (see (11) ) together with the estimates (27) for $z_{h}$ in Lemma 3. Let us next bound $\left\|A^{-1} \Pi \rho_{h, H}^{1}\right\|_{0}$. We will use the decomposition

$$
\rho_{h, H}^{1}=\left(\left(u-u_{H}\right) \cdot \nabla\right)\left(v_{h}-u\right)+\left(\left(u-u_{H}\right) \cdot \nabla\right) u .
$$

Then, we obtain

$$
\begin{aligned}
\left\|A^{-1} \Pi \rho_{h, H}^{1}\right\|_{0}= & \left\|A^{-1} \Pi\left(\left(\left(u_{H}-u\right) \cdot \nabla\right)\left(v_{h}-u\right)\right)\right\|_{0} \\
& +\left\|A^{-1} \Pi\left(\left(\left(u_{H}-u\right) \cdot \nabla\right) u\right)\right\|_{0} .
\end{aligned}
$$

To bound the second term in (41) we apply (33) from Lemma 5 to get

$$
\left\|A^{-1} \Pi\left(\left(\left(u_{H}-u\right) \cdot \nabla\right) u\right)\right\|_{0} \leq C\left\|u_{H}-u\right\|_{-1} .
$$


Applying then (24) together with (12) and (27) we get

$$
\begin{aligned}
\left\|u_{H}-u\right\|_{-1} & \leq\left\|u_{H}-v_{H}\right\|_{0}+\left\|v_{H}-u\right\|_{-1} \\
& \leq \frac{C}{t^{(r-2) / 2}}|\log (H)| H^{r+1}+\frac{C}{t^{(r-2) / 2}} H^{r+1} .
\end{aligned}
$$

To bound the first term in (41) we argue by duality, using (5), we get

$$
\begin{aligned}
& \left\|A^{-1} \Pi\left(\left(\left(u_{H}-u\right) \cdot \nabla\right)\left(v_{h}-u\right)\right)\right\|_{0} \\
& =\sup _{\|\phi\|_{0}=1}\left(\left(\left(u_{H}-u\right) \cdot \nabla\right)\left(v_{h}-u\right), A^{-1} \Pi \phi\right) \\
& \quad \leq \sup _{\|\phi\|_{0}=1}\left\|u_{H}-u\right\|_{0}\left\|v_{h}-u\right\|_{1}\left\|A^{-1} \Pi \phi\right\|_{\infty} \\
& \quad \leq \sup _{\|\phi\|_{0}=1} C\left\|u_{H}-u\right\|_{0}\left\|v_{h}-u\right\|_{1}\left\|A^{-1} \Pi \phi\right\|_{2} \leq C\left\|u_{H}-u\right\|_{0}\left\|v_{h}-u\right\|_{1} .
\end{aligned}
$$

Now, in view of the case $r=2$ in (25) and using again (11) and (27) we conclude

$$
\left\|A^{-1} \Pi\left(\left(\left(u_{H}-u\right) \cdot \nabla\right)\left(v_{h}-u\right)\right)\right\|_{0} \leq \frac{C}{t^{(r-2) / 2}} H^{2} h^{r-1} .
$$

Finally, to bound $\left\|A^{-1} \Pi \rho_{h, H}^{2}\right\|_{0}$ we apply again (33) to bound this norm in terms of $\left\|v_{h}-u\right\|_{-1}$ which, as we shown in (42), is bounded by $C t^{(2-r) / 2} h^{r+1}$.

Lemma 7 Let $(u, p)$ be the solution of (11)-(2). There exists a positive constant $C$ such that the following inequalities hold for $r=3,4$ :

$$
\begin{aligned}
\left\|\rho_{h, H}\right\|_{-1} & \leq \frac{C}{t^{(r-1) / 2}}|\log (H)| H^{r+1}+\frac{C}{t^{(r-2) / 2}} h^{r}, \quad t \in(0, T], \\
\left\|\rho_{h, H}\right\|_{-1} & \leq \frac{C}{t^{1 / 2}} H^{3}, \quad t \in(0, T] .
\end{aligned}
$$

Proof The proof is very similar to the one of the previous lemma. We will prove (43) since the proof of (44) is completely analogous and yet easier. We use the decomposition (37).

For $\rho_{h, H}^{2}$ we apply (30) to get

$$
\left\|\rho_{h, H}^{2}\right\|_{-1} \leq C\left\|v_{h}-u\right\|_{0} \leq C \frac{h^{r}}{t^{(r-2) / 2}},
$$


where we have applied (11) and (27) in the last inequality. For $\rho_{h, H}^{1}$ we will use the decomposition (40). For the first term in (40) using (5) we have

$$
\begin{aligned}
\left\|\left(\left(u_{H}-u\right) \cdot \nabla\right)\left(v_{h}-u\right)\right\|_{-1} & =\sup _{\|\phi\|_{1}=1}\left(\left(\left(u_{H}-u\right) \cdot \nabla\right)\left(v_{h}-u\right), \phi\right) \\
& \leq \sup _{\|\phi\|_{1}=1}\left\|u_{H}-u\right\|_{L^{2 d}}\left\|v_{h}-u\right\|_{1}\|\phi\|_{L^{2 d /(d-1)}} \\
& \leq C\left\|u_{H}-u\right\|_{1}\left\|v_{h}-u\right\|_{1} \leq C \frac{H^{r-1}}{t^{(r-2) / 2}} \frac{h^{2}}{t^{1 / 2}}
\end{aligned}
$$

where we have applied (25) and (11) and (27) in the last inequality. Finally, for the second term using (5), (7) and (42) we obtain

$$
\begin{aligned}
\left\|\left(\left(u_{H}-u\right) \cdot \nabla\right) u\right\|_{-1} & =\sup _{\|\phi\|_{1}=1}\left(\left(\left(u_{H}-u\right) \cdot \nabla\right) u, \phi\right) \\
& \leq \sup _{\|\phi\|_{1}=1}\left\|u_{H}-u\right\|_{-1}\|\phi \nabla u\|_{1} \\
& \leq \sup _{\|\phi\|_{1}=1}\left\|u_{H}-u\right\|_{-1}\left(\|\nabla u\|_{W^{1,2 d /(d-1)}}\|\phi\|_{L^{2 d}}\right. \\
& \left.+\|\nabla u\|_{\infty}\|\phi\|_{1}\right) \\
& \leq \frac{C}{t^{(r-2) / 2}}|\log (H)| H^{r+1}\|u\|_{3} \leq \frac{C}{t^{(r-1) / 2}}|\log (H)| H^{r+1} .
\end{aligned}
$$

Lemma 8 Let $(u, p)$ be the solution of (11)-(2). Then there exists a positive constant $C$ such that the discrete velocity $v_{h}$ defined by (35) and the approximation to $u$ over the finer grid, $\tilde{u}_{h}$ satisfy the following bound:

$$
\left\|A_{h}^{l / 2}\left(v_{h}(t)-\tilde{u}_{h}(t)\right)\right\|_{0} \leq C H^{3-l}, \quad r \geq 3, \quad l=0,1, \quad t \in(0, T] .
$$

Proof Let us consider $y_{h}(t)=A_{h}^{l / 2} e_{h}(t)$. From (36) it follows that $y_{h}(t)=\int_{0}^{t} e^{-(t-s) A_{h}} A_{h}^{l / 2} \Pi_{h}\left(\left(u_{H} \cdot \nabla\right) e_{h}\right) d s+\int_{0}^{t} e^{-(t-s) A_{h}} A_{h}^{l / 2} \Pi_{h} \rho_{h, H}(s) d s$.

Applying (15), and taking into account that as a consequence of (29) and (32) we have $\left\|A_{h}^{(-1+l) / 2} \Pi_{h}\left(\left(u_{H} \cdot \nabla\right) e_{h}\right)\right\|_{0} \leq C\left\|A_{h}^{l / 2} e_{h}\right\|_{0}$, it follows that

$$
\left\|y_{h}(t)\right\|_{0} \leq \int_{0}^{t} \frac{C}{\sqrt{t-s}}\left\|y_{h}\right\|_{0} d s+\left\|\int_{0}^{t} e^{-(t-s) A_{h}} A_{h}^{l / 2} \Pi_{h} \rho_{h, H}(s) d s\right\|,
$$


so that a generalized Gronwall lemma [22, pp. 188-189] allow us to write

$$
\max _{0 \leq t \leq T}\left\|y_{h}(t)\right\|_{0} \leq C \max _{0 \leq t \leq T}\left\|\int_{0}^{t} e^{-(t-s) A_{h}} A_{h}^{l / 2} \Pi_{h} \rho_{h, H}(s) d s\right\|_{0} .
$$

Using (16) we obtain

$$
\max _{0 \leq t \leq T}\left\|y_{h}(t)\right\|_{0} \leq C B\left(\frac{1}{2}, \frac{1}{2}\right) \max _{0 \leq s \leq T} s^{1 / 2}\left\|A_{h}^{(-1+l) / 2} \Pi_{h} \rho_{h, H}\right\|_{0} .
$$

To conclude we apply (13) and (44) from Lemma 7 in the case $l=0$, and (38) and (39) in the case $l=1$.

Lemma 9 Let $(u, p)$ be the solution of (11)-(2). Then, there exists a positive constant $C$ such that the discrete velocity $v_{h}$ defined by (35) and the approximation to $u$ over the finer grid, $\tilde{u}_{h}$ satisfy the following bound:

$$
\left\|A_{h}^{-1 / 2}\left(v_{h}(t)-\tilde{u}_{h}(t)\right)\right\|_{0} \leq C|\log (H)| H^{4}, \quad r \geq 3, \quad t \in(0, T] .
$$

Proof The proof follows the steps of the proof of Lemma 4.6 in [14]. Let us consider $y_{h}(t)=A_{h}^{-1 / 2} e_{h}(t)$. From (36) it follows that

$y_{h}(t)=\int_{0}^{t} e^{-(t-s) A_{h}} A_{h}^{-1 / 2} \Pi_{h}\left(\left(u_{H} \cdot \nabla\right) e_{h}\right) d s+\int_{0}^{t} e^{-(t-s) A_{h}} A_{h}^{-1 / 2} \Pi_{h} \rho_{h, H}(s) d s$.

Applying (15) we have that

$$
\begin{aligned}
\left\|y_{h}(t)\right\|_{0} \leq \int_{0}^{t} & \frac{C}{\sqrt{t-s}}\left\|A_{h}^{-1} \Pi_{h}\left(\left(u_{H} \cdot \nabla\right) e_{h}\right)\right\|_{0} d s \\
& +\left\|\int_{0}^{t} e^{-(t-s) A_{h}} A_{h}^{-1 / 2} \Pi_{h} \rho_{h, H}(s) d s\right\|_{0}
\end{aligned}
$$

Let us now bound $\left\|A_{h}^{-1} \Pi_{h}\left(\left(u_{H} \cdot \nabla\right) e_{h}\right)\right\|_{0}$. We will argue as in the proof of [14, (4.23) Lemma 4.4]. Let us first observe that $h^{2}\left\|e_{h}\right\|_{1} \leq C\left\|A_{h}^{-1 / 2} e_{h}\right\|_{0}$. Using (14) we get

$$
\begin{aligned}
\left\|A_{h}^{-1} \Pi_{h}\left(\left(u_{H} \cdot \nabla\right) e_{h}\right)\right\|_{0} & \leq C h^{2}\left\|\left(u_{H} \cdot \nabla\right) e_{h}\right\|_{0}+\left\|A^{-1} \Pi\left(\left(u_{H} \cdot \nabla\right) e_{h}\right)\right\|_{0} \\
& \leq C h^{2}\left\|e_{h}\right\|_{1}+\left\|A^{-1} \Pi\left(\left(u_{H} \cdot \nabla\right) e_{h}\right)\right\|_{0} \\
& \leq C\left\|A_{h}^{-1 / 2} e_{h}\right\|_{0}+\left\|A^{-1} \Pi\left(\left(u_{H} \cdot \nabla\right) e_{h}\right)\right\|_{0} .
\end{aligned}
$$


Let us now bound the second term on the right hand side above. We write $\left\|A^{-1} \Pi\left(\left(u_{H} \cdot \nabla\right) e_{h}\right)\right\|_{0} \leq\left\|A^{-1} \Pi\left(\left(\left(u_{H}-u\right) \cdot \nabla\right) e_{h}\right)\right\|_{0}+\left\|A^{-1} \Pi\left((u \cdot \nabla) e_{h}\right)\right\|_{0}$.

For the first term arguing by duality we get

$$
\left\|A^{-1} \Pi\left(\left(\left(u_{H}-u\right) \cdot \nabla\right) e_{h}\right)\right\|_{0} \leq C\left\|u_{H}-u\right\|_{0}\left\|e_{h}\right\|_{1} \leq C H^{2}\left\|e_{h}\right\|_{1} .
$$

For the second one, arguing again by duality and integrating by parts, we get

$$
\left\|A^{-1} \Pi\left((u \cdot \nabla) e_{h}\right)\right\|_{0} \leq C\left\|e_{h}\right\|_{-1}\|u\|_{2} \leq C\left\|y_{h}\right\|_{0} .
$$

We finally obtain

$$
\left\|A_{h}^{-1} \Pi_{h}\left(\left(u_{H} \cdot \nabla\right) e_{h}\right)\right\|_{0} \leq C\left\|y_{h}\right\|_{0}+C H^{2}\left\|e_{h}\right\|_{1} .
$$

Going back to (47) we obtain

$$
\begin{gathered}
\left\|y_{h}(t)\right\|_{0} \leq \int_{0}^{t} \frac{C}{\sqrt{t-s}}\left\|y_{h}\right\|_{0} d s+\left\|\int_{0}^{t} e^{-(t-s) A_{h}} A_{h}^{-1 / 2} \Pi_{h} \rho_{h, H}(s) d s\right\|_{0} \\
+C t^{1 / 2} H^{2} \max _{0 \leq s \leq t}\left\|e_{h}(s)\right\|_{1}
\end{gathered}
$$

so that a generalized Gronwall lemma [22, pp. 188-189] allow us to write

$$
\max _{0 \leq t \leq T}\left\|y_{h}(t)\right\|_{0} \leq C\left(\max _{0 \leq t \leq T}\left\|\int_{0}^{t} e^{-(t-s) A_{h}} A_{h}^{-1 / 2} \Pi_{h} \rho_{h, H} d s\right\|_{0}+H^{2} \max _{0 \leq t \leq T}\left\|e_{h}\right\|_{1}\right) .
$$

Using (16) we obtain

$$
\max _{0 \leq t \leq T}\left\|y_{h}(t)\right\|_{0} \leq C\left(B\left(\frac{1}{2}, \frac{1}{2}\right) \max _{0 \leq s \leq T} s^{1 / 2}\left\|A_{h}^{-1} \Pi_{h} \rho_{h, H}\right\|_{0}+H^{2} \max _{0 \leq t \leq T}\left\|e_{h}\right\|_{1}\right),
$$

where, by applying Lemmas 6 and 8 the proof is finished.

The proof of the following theorem follows the steps of the proof of [14, Theorem 4.7].

Theorem 1 Let $(u, p)$ be the solution of (11)-(2). There exists a positive constant $C$ such that the discrete velocity $v_{h}$ defined by (35) and the approximation to $u$ over the finer grid, $\tilde{u}_{h}$, satisfy the following bound:

$$
\left\|v_{h}(t)-\tilde{u}_{h}(t)\right\|_{0} \leq \frac{C}{t^{1 / 2}}|\log (h)|\left(|\log (H)| H^{4}\right), \quad t \in(0, T], \quad r \geq 3 .
$$


Proof Let us consider $y_{h}(t)=t^{1 / 2} e_{h}(t)$. From (36) and an easy calculation we get

$$
\dot{y}_{h}+A_{h} y_{h}+t^{1 / 2} \Pi_{h}\left(u_{H} \cdot \nabla e_{h}\right)=t^{1 / 2} \Pi_{h} \rho_{h, H}+\frac{1}{2 t^{1 / 2}} e_{h} .
$$

Then,

$$
\begin{aligned}
y_{h}(t)=\int_{0}^{t} e^{-A_{h}(t-s)} s^{1 / 2} \Pi_{h}\left(u_{H}\right. & \left.\cdot \nabla e_{h}\right) d s \\
& +\int_{0}^{t} e^{-A_{h}(t-s)}\left(s^{1 / 2} \Pi_{h} \rho_{h, H}+\frac{1}{2 s^{1 / 2}} e_{h}\right) d s .
\end{aligned}
$$

Applying (32) we get

$$
\left.\| A_{h}^{-1 / 2} \Pi_{h}\left(u_{H} \cdot \nabla\right) e_{h}\right)\left\|_{0} \leq C\right\| e_{h} \|_{0} .
$$

Then, using (15) we obtain

$$
\left\|\int_{0}^{t} e^{-A_{h}(t-s)} s^{1 / 2} \Pi_{h}\left(u_{H} \cdot \nabla e_{h}\right) d s\right\|_{0} \leq C \int_{0}^{t} \frac{\left\|y_{h}\right\|_{0}}{\sqrt{t-s}} d s .
$$

Applying a generalized Gronwall lemma [22, pp. 188-189], it follows that

$$
\begin{aligned}
\max _{0 \leq t \leq T}\left\|y_{h}(t)\right\|_{0} \leq & C\left(\max _{0 \leq t \leq T}\left\|\int_{0}^{t} e^{-A_{h}(t-s)} s^{1 / 2} \Pi_{h} \rho_{h, H} d s\right\|_{0}\right. \\
& \left.+\max _{0 \leq s \leq t}\left\|\int_{0}^{t} e^{-A_{h}(t-s)} \frac{e_{h}}{s^{1 / 2}} d s\right\|_{0}\right) .
\end{aligned}
$$

Applying now Lemma 2 and (16) we have

$$
\begin{aligned}
\max _{0 \leq t \leq T}\left\|y_{h}(t)\right\|_{0} \leq & C\left(|\log (h)| \max _{0 \leq t \leq T}\left\|s^{1 / 2} A_{h}^{-1} \Pi_{h} \rho_{h, H}(s)\right\|_{0}\right. \\
& \left.+B\left(\frac{1}{2}, \frac{1}{2}\right) \max _{0 \leq t \leq T}\left\|A_{h}^{-1 / 2} e_{h}(s)\right\|_{0}\right)
\end{aligned}
$$

where Lemmas 6 and 9 finish the proof.

Theorem 2 Let $(u, p)$ be the solution of (11)-(2). There exists a positive constant $C$ such that the discrete velocity $v_{h}$ defined by (35) and the approximation to $u$ over the finer grid, $\tilde{u}_{h}$, satisfy the following bound for $r \geq 3$

$$
\left\|v_{h}(t)-\tilde{u}_{h}(t)\right\|_{1} \leq \frac{C}{t}|\log (h)|\left(|\log (H)| H^{4}+T^{1 / 2} h^{3}\right), \quad t \in(0, T] .
$$


Proof Let us define $y_{h}(t)=t A_{h}^{1 / 2} e_{h}(t)$, where $e_{h}(t)=v_{h}(t)-\tilde{u}_{h}(t)$. Arguing exactly as in the proof of Theorem 1, instead of (153) we now arrive at

$$
\begin{aligned}
\max _{0 \leq t \leq T}\left\|y_{h}(t)\right\|_{0} \leq & C\left(\max _{0 \leq t \leq T}\left\|\int_{0}^{t} e^{-A_{h}(t-s)} s A_{h}^{1 / 2} \Pi_{h} \rho_{h, H} d s\right\|_{0}\right. \\
& \left.+\max _{0 \leq t \leq T}\left\|\int_{0}^{t} e^{-A_{h}(t-s)} A_{h}^{1 / 2} e_{h} d s\right\|_{0}\right) .
\end{aligned}
$$

Applying now Lemma 2 we get

$$
\max _{0 \leq t \leq T}\left\|y_{h}(t)\right\|_{0} \leq C|\log (h)|\left(\max _{0 \leq t \leq T}\left\|s A_{h}^{-1 / 2} \Pi_{h} \rho_{h, H}\right\|_{0}+\max _{0 \leq t \leq T}\left\|A_{h}^{-1 / 2} e_{h}\right\|_{0}\right),
$$

where Lemmas 7 and 9 finish the proof.

Lemma 10 Let $(u, p)$ be the solution of (1)-(2). Then there exists a positive constant $C$ such that the discrete velocity $v_{h}$ defined by (35) and the approximation to $u$ over the finer grid, $\tilde{u}_{h}$ satisfy the following bound:

$$
\left\|A_{h}^{-1}\left(v_{h}(t)-\tilde{u}_{h}(t)\right)\right\|_{0} \leq C H^{5}, \quad r \geq 4, \quad t \in(0, T] .
$$

Proof Let us consider $y_{h}(t)=A_{h}^{-1} e_{h}(t)$. From (36) it follows that $y_{h}(t)=\int_{0}^{t} e^{-(t-s) A_{h}} A_{h}^{-1} \Pi_{h}\left(\left(u_{H} \cdot \nabla\right) e_{h}\right) d s+\int_{0}^{t} e^{-(t-s) A_{h}} A_{h}^{-1} \Pi_{h} \rho_{h, H}(s) d s$.

We first observe that arguing exactly as in the proof of [14, Lemma 4.13] we get

$$
\begin{aligned}
\left\|e^{-(t-s) A_{h}} A_{h}^{-1} \Pi_{h}\left(\left(u_{H} \cdot \nabla\right) e_{h}\right)\right\|_{0} \leq & C\left(\frac{1}{\sqrt{t-s}}+\frac{1}{\sqrt{s}}\right)\left\|A_{h}^{-1} e_{h}\right\|_{0} \\
+ & C \frac{H^{3}}{\sqrt{s}}\left\|e_{h}\right\|_{1} .
\end{aligned}
$$

Then,

$$
\begin{aligned}
\left\|y_{h}(t)\right\|_{0} \leq & C \int_{0}^{t}\left(\frac{1}{\sqrt{t-s}}+\frac{1}{\sqrt{s}}\right)\left\|y_{h}(s)\right\|_{0} d s \\
& +\left\|\int_{0}^{t} e^{-(t-s) A_{h}} A_{h}^{-1} \Pi_{h} \rho_{h, H}(s) d s\right\|+C t^{1 / 2} H^{3} \max _{0 \leq s \leq t}\left\|e_{h}(s)\right\|_{1}
\end{aligned}
$$


Applying now [14, Lemma 4.9] we get

$$
\begin{aligned}
\max _{0 \leq t \leq T}\left\|y_{h}(t)\right\|_{0} \leq & C \max _{0 \leq t \leq T}\left\|\int_{0}^{t} e^{-(t-s) A_{h}} A_{h}^{-1} \Pi_{h} \rho_{h, H}(s) d s\right\| \\
& +C H^{3} \max _{0 \leq t \leq T}\left\|e_{h}(t)\right\|_{1} .
\end{aligned}
$$

For the second term on the right-hand-side above we apply Lemma 8 , For the first one we use the decomposition

$$
\rho_{h, H}=\rho_{h, H}^{1}+\rho_{h, H}^{2}, \quad \rho_{h, H}^{1}=\left(\left(u_{H}-u\right) \cdot \nabla\left(v_{h}-u\right)\right)+\left(\left(u_{H}-u\right) \cdot \nabla u\right),
$$

We now argue exactly as in [14, (4.60) in Lemma 4.14], replacing one of the occurrences of $z$ there by $u-u_{H}$ and making use of (25) and (26) with $h$ replaced by $H$. This will allow us to obtain

$$
\max _{0 \leq t \leq T}\left\|\int_{0}^{t} e^{-(t-s) A_{h}} A_{h}^{-1} \Pi_{h} \rho_{h, H}(s) d s\right\| \leq C H^{5},
$$

which concludes the proof.

Theorem 3 Let $(u, p)$ be the solution of (1)-(2). There exists a positive constant $C$ such that the discrete velocity $v_{h}$ defined by (35) and the approximation to $u$ over the finer grid, $\tilde{u}_{h}$, satisfy the following bound:

$$
\left\|v_{h}(t)-\tilde{u}_{h}(t)\right\|_{0} \leq \frac{C}{t}|\log (h)|\left(|\log (H)| H^{5}\right), \quad t \in(0, T], \quad r \geq 4 .
$$

Proof Let us define $y_{h}(t)=t e_{h}(t)$, where $e_{h}(t)=v_{h}(t)-\tilde{u}_{h}(t)$. Arguing as in the proof of Theorem 1, instead of (53) we now arrive at

$$
\begin{aligned}
\max _{0 \leq t \leq T}\left\|y_{h}(t)\right\|_{0} \leq & C\left(\max _{0 \leq t \leq T}\left\|\int_{0}^{t} e^{-A_{h}(t-s)} s \Pi_{h} \rho_{h, H} d s\right\|_{0}\right. \\
& \left.+\max _{0 \leq t \leq T}\left\|\int_{0}^{t}\right\| e^{-A_{h}(t-s)} e_{h} d s \|_{0}\right) .
\end{aligned}
$$

As in the proof of Theorem 2, applying now Lemma 2 to both terms on the right-hand side above we get

$$
\max _{0 \leq t \leq T}\left\|y_{h}(t)\right\|_{0} \leq C|\log (h)|\left(\max _{0 \leq t \leq T}\left\|s A_{h}^{-1} \Pi_{h} \rho_{h, H}\right\|_{0}+\max _{0 \leq t \leq T}\left\|A_{h}^{-1} e_{h}\right\|_{0}\right) .
$$

where now Lemmas 6 and 10 finish the proof. 
Lemma 11 Let $(u, p)$ be the solution of (1)-(2). Then there exists a positive constant $C$ such that the discrete velocity $v_{h}$ defined by (35) and the approximation to $u$ over the finer grid, $\tilde{u}_{h}$ satisfy the following bound:

$$
\left\|A_{h}^{-1 / 2}\left(v_{h}(t)-\tilde{u}_{h}(t)\right)\right\|_{0} \leq \frac{C}{t^{1 / 2}}|\log (h)| H^{5}, \quad r \geq 4, \quad t \in(0, T] .
$$

Proof Setting $y_{h}(t)=t^{1 / 2} A_{h}^{-1 / 2} e_{h}(t)$ and arguing exactly as in the proof of Lemma 9, instead of (49) we now obtain

$$
\begin{aligned}
\left\|y_{h}(t)\right\|_{0} \leq & \int_{0}^{t} \frac{C}{\sqrt{t-s}}\left\|y_{h}\right\|_{0} d s+\left\|\int_{0}^{t} e^{-(t-s) A_{h}} s^{1 / 2} A_{h}^{-1 / 2} \Pi_{h} \rho_{h, H}(s) d s\right\|_{0} \\
& +\left\|\int_{0}^{t} e^{-(t-s) A_{h}} A_{h}^{-1 / 2} \frac{e_{h}(s)}{2 s^{1 / 2}} d s\right\|_{0}+C H^{2} \max _{0 \leq s \leq t} s^{1 / 2}\left\|e_{h}(s)\right\|_{1},
\end{aligned}
$$

so that a generalized Gronwall lemma [22, pp. 188-189] allow us to write

$$
\begin{aligned}
\max _{0 \leq t \leq T}\left\|y_{h}(t)\right\|_{0} \leq C \max _{0 \leq t \leq T}\left\|\int_{0}^{t} e^{-(t-s) A_{h}} s^{1 / 2} A_{h}^{-1 / 2} \Pi_{h} \rho_{h, H}(s) d s\right\|_{0} \\
+\max _{0 \leq t \leq T}\left\|\int_{0}^{t} e^{-(t-s) A_{h}} A_{h}^{-1 / 2} \frac{e_{h}(s)}{2 s^{1 / 2}} d s\right\|_{0}+C H^{2} \max _{0 \leq t \leq T} t^{1 / 2}\left\|e_{h}(t)\right\|_{1} .
\end{aligned}
$$

Using (16) we obtain

$$
\begin{aligned}
\max _{0 \leq t \leq T}\left\|y_{h}(t)\right\|_{0} \leq & C B\left(\frac{1}{2}, \frac{1}{2}\right)\left(\max _{0 \leq s \leq T}\left\|s A_{h}^{-1} \Pi_{h} \rho_{h, H}\right\|_{0}+\max _{0 \leq s \leq T}\left\|A_{h}^{-1} e_{h}\right\|_{0}\right) \\
& +C H^{2} \max _{0 \leq t \leq T} t^{1 / 2}\left\|e_{h}\right\|_{1} .
\end{aligned}
$$

For the first two terms on the right-hand-side above we apply Lemmas 6 and 10 respectively. For the last term we observe that denoting by $y_{h}(t)=$ $t^{1 / 2} A_{h}^{1 / 2} e_{h}$ and arguing as in Theorem 1 we get

$$
\begin{aligned}
\max _{0 \leq t \leq T}\left\|y_{h}(t)\right\|_{0} \leq & C\left(|\log (h)| \max _{0 \leq t \leq T}\left\|t^{1 / 2} A_{h}^{-1 / 2} \Pi_{h} \rho_{h, H}\right\|_{0}\right. \\
& \left.+B\left(\frac{1}{2}, \frac{1}{2}\right) \max _{0 \leq t \leq T}\left\|e_{h}\right\|_{0}\right)
\end{aligned}
$$

so that applying now (13) and (44) to bound the first term on the right-hand side above, and the case $l=0$ in Lemma 8 for the second one, the proof is completed. 
Theorem 4 Let $(u, p)$ be the solution of (1)-(2). There exists a positive constant $C$ such that the discrete velocity $v_{h}$ defined by (35) and the approximation to $u$ over the finer grid, $\tilde{u}_{h}$, satisfy the following bound for $r \geq 4$ :

$$
\left\|v_{h}(t)-\tilde{u}_{h}(t)\right\|_{1} \leq \frac{C}{t^{3 / 2}}|\log (h)|\left(|\log (h)| H^{5}+T^{1 / 2} h^{4}\right), \quad t \in(0, T] .
$$

Proof Let $y_{h}(t)=t^{3 / 2} A_{h}^{1 / 2} e_{h}$ and argue as in the proof of Theorem 2 to get

$\left\|y_{h}(t)\right\|_{0} \leq C|\log (h)|\left(\max _{0 \leq t \leq T}\left\|t^{3 / 2} A_{h}^{-1 / 2} \Pi_{h} \rho_{h, H}\right\|_{0}+\max _{0 \leq t \leq T}\left\|t^{1 / 2} A_{h}^{-1 / 2} e_{h}\right\|_{0}\right)$,

for $t \in(0, T]$. To bound the first term on the right-hand side above we apply (13) and (43), and for the second one we apply Lemma 11.

We now summarize the main results of the section in the following theorem.

Theorem 5 Let $(u, p)$ be the solution of (11)-(2). There exists a positive constant $C$ such that the approximation to $u$ over the finer grid, $\tilde{u}_{h}$, satisfy the following bounds for $r=3,4$ and $t \in(0, T]$ :

$$
\begin{aligned}
& \left\|u(t)-\tilde{u}_{h}(t)\right\|_{0} \leq \frac{C}{t^{(r-2) / 2}}|\log (h)||\log (H)| H^{r+1}+\frac{C}{t^{(r-2) / 2}} h^{r} . \\
& \left\|u(t)-\tilde{u}_{h}(t)\right\|_{1} \leq \frac{C}{t^{(r-1) / 2}}|\log (h)|\left(|\log (h)| H^{r+1}+T^{1 / 2} h^{r}\right)+\frac{C}{t^{(r-2) / 2}} h^{r-1},
\end{aligned}
$$

where in the last inequality we can replace the second $|\log (h)|$ by $|\log (H)|$ in the case $r=3$.

Proof We use the decomposition $u-\tilde{u}_{h}=\left(u-v_{h}\right)+\left(v_{h}-\tilde{u}_{h}\right)$. To bound the first term we apply (11) and (27) while for the second we apply Theorems 1, 4 .

Now, we get the error bounds for the pressure. We begin with some error estimates for the time derivative of $v_{h}-\tilde{u}_{h}$.

Lemma 12 Let $(u, p)$ be the solution of (1)-(2). Then there exists a positive constant $C$ such that the discrete velocity $v_{h}$ defined by (35) and the approximation to $u$ over the finer grid, $\tilde{u}_{h}$ satisfy the following bound for $r=3,4$ :

$$
\left\|\dot{v}_{h}(t)-\dot{\tilde{u}}_{h}(t)\right\|_{-1} \leq \frac{C}{t^{(r-1) / 2}}|\log (h)|\left(|\log (h)| H^{r+1}+h^{r}\right), t \in(0, T] .
$$

In the case $r=3$ the second $\log (h)$ can be replaced by $\log (H)$. 
Proof Using (36) and taking into account that $\left\|\dot{e}_{h}\right\|_{-1} \leq C\left\|A_{h}^{-1 / 2} \dot{e}_{h}\right\|_{0}$ we obtain

$$
\begin{aligned}
\left\|\dot{e}_{h}\right\|_{-1} & \leq\left\|A_{h}^{1 / 2} e_{h}\right\|_{0}+\left\|A_{h}^{-1 / 2} \Pi_{h}\left(\left(u_{H} \cdot \nabla\right) e_{h}+\rho_{h, H}\right)\right\|_{0} \\
& \leq\left\|e_{h}\right\|_{1}+C\left\|e_{h}\right\|_{0}+\left\|A_{h}^{-1 / 2} \Pi_{h} \rho_{h, H}\right\|_{0},
\end{aligned}
$$

after using (52) in the last inequality. Applying now Theorems 144 together with (13) and (43) we reach (59).

The following Lemma is proved in [14, Corollary 4.19] and Proposition 3.1 in [23] and [24] (see also [6] and [15]).

Lemma 13 Let $(u, p)$ be the solution of (1)-(2) and let $\left(u_{h}, p_{h}\right)$ and $\left(v_{h}, g_{h}\right)$ the approximations defined in (17)-(18) and (21)-(22), respectively. Then, the following bound holds for $r=2,3,4$

$$
\begin{aligned}
& \left\|p_{h}(t)-p(t)\right\|_{L^{2} / \mathbb{R}} \leq \frac{C}{t^{(r-2) / 2}} h^{r-1}, \quad t \in(0, T], \\
& \left\|g_{h}(t)-p(t)\right\|_{L^{2} / \mathbb{R}} \leq \frac{C}{t^{(r-2) / 2}} h^{r-1}, \quad t \in(0, T] .
\end{aligned}
$$

Theorem 6 Let $(u, p)$ be the solution of (11)-(2). There exists a positive constant $C$ such that the approximation to $p$ over the finer grid, $\tilde{p}_{h}$, satisfies the following bound for $t \in(0, T]$ and $r=3,4$ :

$$
\left\|\tilde{p}_{h}(t)-p(t)\right\|_{L^{2} / \mathbb{R}} \leq \frac{C}{t^{(r-2) / 2}} h^{r-1}+\frac{C}{t^{(r-1) / 2}}|\log (h)|\left(|\log (h)| H^{r+1}+h^{r}\right) .
$$

In the case $r=3$ the second $\log (h)$ can be replaced by $\log (H)$.

Proof We use the decomposition

$$
\left\|\tilde{p}_{h}-p\right\|_{L^{2} / \mathbb{R}} \leq\left\|\tilde{p}_{h}-g_{h}\right\|_{L^{2} / \mathbb{R}}+\left\|g_{h}-p\right\|_{L^{2} / \mathbb{R}} .
$$

To bound the second term on the right-hand-side above we apply (61). For the first one subtracting (21) from (19) and applying the inf-sup condition (10) we obtain

$$
\beta\left\|\tilde{p}_{h}-g_{h}\right\|_{L^{2} / \mathbb{R}} \leq\left\|\tilde{u}_{h}-v_{h}\right\|_{1}+\left\|\left(u_{H} \cdot \nabla\right) e_{h}\right\|_{-1}+\left\|\rho_{h, H}\right\|_{-1}+\left\|\dot{e}_{h}\right\|_{-1} .
$$

We first observe that applying (30) we get $\left\|\left(u_{H} \cdot \nabla\right) e_{h}\right\|_{-1} \leq C\left\|e_{h}\right\|_{0}$. To bound $\left\|\rho_{h, H}\right\|_{-1}$ we apply (43). Now, the proof concludes applying Theorems 14 together with (59). 
We state in the following theorem the results that can be obtained for the mini-element.

Theorem 7 Let $(u, p)$ be the solution of (11)-(2). There exists a positive constant $C$ such that the approximations over the finer grid computed using the mini-element, $\left(\tilde{u}_{h}, \tilde{p}_{h}\right)$, satisfy the following bounds for $t \in(0, T]$ :

$$
\begin{aligned}
\|\tilde{u}(t)-u(t)\|_{0} & \leq C H^{2}+C h^{2}, \\
\|\tilde{u}(t)-u(t)\|_{1} & \leq C|\log (h)| H^{2}+C h, \\
\left\|\tilde{p}_{h}(t)-p(t)\right\|_{L^{2} / \mathbb{R}} & \leq C|\log (h)| H^{2}+C h .
\end{aligned}
$$

\section{Semi-discretization in space. The second two- grid algorithm.}

As in the previous section we will concentrate on the approximations obtained with the Hood-Taylor mixed finite element and $r=3$ or $r=4$ and we will state at the end of the section the results that can be obtained for the mini-element method with a much simpler analysis.

In the second algorithm we consider, the first level, as before, is given by the standard mixed finite-element approximation to (11)-(2), that is, the solution of (17)-(18) with initial condition $u_{H}(0)=\Pi_{H}\left(u_{0}\right)$. In the second level we solve a linearized problem on a finer grid and given $\tilde{u}_{h}(0)=\Pi_{h} u_{0}$, we compute $\tilde{u}_{h}(t) \in X_{h, r}$ and $\tilde{p}_{h}(t) \in Q_{h, r-1}, t \in(0, T]$, satisfying, for all $\phi_{h} \in X_{h, r}$ and $\psi_{h} \in Q_{h, r-1}$

$$
\begin{array}{r}
\left(\dot{\tilde{u}}_{h}, \phi_{h}\right)+\left(\nabla \tilde{u}_{h}, \nabla \phi_{h}\right)+b\left(u_{H}, \tilde{u}_{h}, \phi_{h}\right)+b\left(\tilde{u}_{h}, u_{H}, \phi_{h}\right)+\left(\nabla \tilde{p}_{h}, \phi_{h}\right)= \\
\left(f, \phi_{h}\right)+b\left(u_{H}, u_{H}, \phi_{h}\right),
\end{array}
$$

Observe that the approximation $\tilde{u}_{H}$ is the result of one step of Newton's method for the Galerkin $\left(u_{h}, p_{h}\right)$ approximation in $X_{h, r} \times Q_{h, r-1}$ (equations (17)-(18) with $H$ replaced by $h)$ with $\left(u_{H}, p_{H}\right)$ as initial approximation. For this reason, in this section we study the error $e_{h}=u_{h}-\tilde{u}_{h}$.

It is easy to obtain that

$$
\dot{e}_{h}+A_{h} e_{h}+B_{h}\left(u_{H}, e_{h}\right)+B_{h}\left(e_{h}, u_{H}\right)=\Pi_{h} \rho_{h, H},
$$

where

$$
\rho_{h, H}=-F\left(\epsilon_{h, H}, \epsilon_{h, H}\right),
$$


where, here and in the sequel,

$$
\epsilon_{h, H}=u_{H}-u_{h}
$$

The analysis in this section is closely related to that in the previous section. However, some extra results are needed. We shall use the following two bounds,

$$
\begin{aligned}
\left\|\phi_{h}\right\|_{L^{2 d /(d-1)}} & \leq C\left\|\phi_{h}\right\|_{0}^{1 / 2}\left\|A_{h}^{1 / 2} \phi_{h}\right\|_{0}^{1 / 2}, \quad \forall \phi_{h} \in V_{h, r}, \\
\left\|\phi_{h}\right\|_{L^{\infty}} & \leq C\left\|A_{h}^{1 / 2} \phi_{h}\right\|_{0}^{1 / 2}\left\|A_{h} \phi_{h}\right\|_{0}^{1 / 2}, \quad \forall \phi_{h} \in V_{h, r},
\end{aligned}
$$

which follow from Corollary 4.4 and Lemma 4.4 in [23]. Also we shall use the following two bounds

$$
\begin{aligned}
\left\|A_{h}^{-1 / 2} B_{h}\left(\epsilon_{h, H}, \epsilon_{h, H}\right)\right\|_{0} & \leq C\left\|\epsilon_{h, H}\right\|_{0}^{1 / 2}\left\|\epsilon_{h, H}\right\|_{1}^{3 / 2}, \\
\left\|A_{h}^{-1} B_{h}\left(\epsilon_{h, H}, \epsilon_{h, H}\right)\right\|_{0} & \leq C\left\|\epsilon_{h, H}\right\|_{0}\left\|\epsilon_{h, H}\right\|_{1}
\end{aligned}
$$

with $C$ independent of $h$, and where here and in the sequel $B_{h}\left(v_{h}, w_{h}\right)=$ $\Pi_{h} F\left(u_{h}, w_{h}\right)$. Both are easily obtained by duality arguments, the first one from (65) and the second one from (66). Notice also that as a consequence of (65)-(66) and (15) we have that $\left\|e^{-t A_{h}} \phi_{h}\right\|_{L^{\infty}} \leq C t^{-3 / 4}\left\|\phi_{h}\right\|_{0}$ and $\left\|e^{-t A_{h}} A_{h}^{1 / 2} \phi_{h}\right\|_{L^{2 d /(d-1)}} \leq C t^{-3 / 4}\left\|\phi_{h}\right\|_{0}$ so that by using duality arguments together with these two inequalities the following two bounds easily follow

$$
\begin{aligned}
\left\|e^{-(t-s) A_{h}} B_{h}\left(\epsilon_{h, H}, \epsilon_{h, H}\right)\right\|_{0} & \leq \frac{C}{(t-s)^{3 / 4}}\left\|\epsilon_{h, H}\right\|_{0}\left\|\epsilon_{h, H}\right\|_{1} . \\
\left\|e^{-(t-s) A_{h}} A_{h}^{1 / 2} B_{h}\left(\epsilon_{h, H}, \epsilon_{h, H}\right)\right\|_{0} & \leq \frac{C}{(t-s)^{3 / 4}}\left\|\epsilon_{h, H}\right\|_{1}^{2} .
\end{aligned}
$$

Lemma 14 There exists a positive constant $C=C\left(M_{2}\right)$ such that

$$
\left\|u-u_{H}\right\|_{L^{\infty}} \leq C H^{1 / 2}
$$

Proof We will use the fact that, due to Lemma 4.3 and 4.4 in [23], and Corollary 4.4 in [23],

$$
\left\|\nabla \Pi_{H} u\right\|_{L^{6}} \leq C\|A u\|_{0}
$$

We write $u-u_{H}=\left(I-\Pi_{H}\right) u+\left(\Pi_{H} u-u_{H}\right)$. Applying (9), we have $\left\|\Pi_{H} u-u_{H}\right\|_{L^{\infty}} \leq C H^{-3 / 2}\left\|\Pi_{h} u-u_{H}\right\|_{0} \leq C H^{1 / 2}$, where in the last inequality we have applied (11) and (25). On the other hand, applying [23, 


$$
\begin{aligned}
\left\|\left(I-\Pi_{H}\right) u\right\|_{L^{\infty}} & \leq C\left\|\left(I-\Pi_{H}\right) u\right\|_{L^{6}}^{1 / 2}\left\|\nabla\left(I-\Pi_{H}\right) u\right\|_{L^{6}}^{1 / 2} \\
& \leq C\left\|\left(I-\Pi_{H}\right) u\right\|_{1}^{1 / 2}\left(\|\nabla u\|_{L^{6}}+\left\|\nabla \Pi_{h} u\right\|_{L^{6}}\right)^{1 / 2} .
\end{aligned}
$$

Now, where, in the last inequality we have applied (5) and [23, Lemma 4.4]. Furthermore, applying (11), (5) and (71) the proof is finished.

Lemma 15 Let $(u, p)$ be the solution of (11)-(2). Then there exists a positive constant $C$ such that the approximations $u_{h}$ and $\tilde{u}_{h}$ to the velocity $u$ over the fine mesh satisfy the following bound:

$$
\left\|A_{h}^{l / 2}\left(u_{h}(t)-\tilde{u}_{h}(t)\right)\right\|_{0} \leq C H^{7 / 2-l}, \quad r \geq 3, \quad l=0,1, \quad t \in(0, T] .
$$

Proof Follow the arguments in the proof of Lemma 8 to obtain (46). Now, for $l=1$ we write $s^{1 / 2}\left\|\Pi_{h} \rho_{h, H}\right\|_{0} \leq C s^{1 / 2}\left\|\epsilon_{h, H}\right\|_{L^{\infty}}\left\|\epsilon_{h, H}\right\|_{1}$, so that applying Lemma 14 and (25) the proof of the case $l=1$ is finished. For $l=0$, applying (67) we have

$$
\begin{aligned}
s^{1 / 2}\left\|A_{h}^{-1 / 2} \Pi_{h} \rho_{h, H}\right\|_{0} & \leq C s^{1 / 2}\left\|\epsilon_{h, H}\right\|_{0}^{1 / 2}\left\|\epsilon_{h, H}\right\|_{1}^{3 / 2} \\
& =C\left(\left\|\epsilon_{h, H}\right\|_{0}\left\|\epsilon_{h, H}\right\|_{1}\right)^{1 / 2}\left\|s^{1 / 2} \epsilon_{h, H}\right\|_{1},
\end{aligned}
$$

so that applying (25) the proof is finished.

Lemma 16 Let $(u, p)$ be the solution of (11)-(2). Then there exists a positive constant $C$ such that the approximations $u_{h}$ and $\tilde{u}_{h}$ to the velocity $u$ over the fine mesh satisfy the following bound:

$$
\left\|A_{h}^{-1}\left(u_{h}(t)-\tilde{u}_{h}(t)\right)\right\|_{0} \leq C H^{5}, \quad r \geq 3, \quad t \in(0, T] .
$$

Proof Follow the arguments in the proof of Lemma 10, but notice that now due to the terms $\left(e_{h} \cdot \nabla\right) u_{H}$ and $\left(\nabla \cdot u_{H}\right) e_{H}$, instead of (55) we have

$$
\begin{aligned}
\left\|e^{-(t-s) A_{h}} A_{h}^{-1} \Pi_{h}\left(\left(u_{H} \cdot \nabla\right) e_{h}\right)\right\|_{0} \leq & C\left(\frac{1}{\sqrt{t-s}}+\frac{1}{\sqrt{s}}\right)\left\|A_{h}^{-1} e_{h}\right\|_{0} \\
& +C\left(\frac{H^{3}}{\sqrt{s}}\left\|e_{h}\right\|_{1}+\frac{H^{2}}{\sqrt{s}}\left\|e_{h}\right\|_{0}\right) .
\end{aligned}
$$

Thus, instead of (56) we arrive at

$$
\begin{aligned}
\max _{0 \leq t \leq T}\left\|y_{h}(t)\right\|_{0} \leq & C \max _{0 \leq t \leq T}\left\|\int_{0}^{t} e^{-(t-s) A_{h}} A_{h}^{-1} \Pi_{h} \rho_{h, H}(s) d s\right\| \\
& +C\left(H^{3} \max _{0 \leq t \leq T}\left\|e_{h}(t)\right\|_{1}+H^{2} \max _{0 \leq t \leq T}\left\|e_{h}(t)\right\|_{0}\right) .
\end{aligned}
$$


Thanks to Lemma 15 we have that the last two terms on the right-hand side above are bounded by $C H^{11 / 2}$. For the first one, applying first (68) and then (26) we conclude that it is also bounded by $C H^{5}$.

Lemma 17 Let $(u, p)$ be the solution of (11)-(2). Then there exists a positive constant $C$ such that the approximations $u_{h}$ and $\tilde{u}_{h}$ to the velocity $u$ over the fine mesh satisfy the following bound:

$$
\left\|A_{h}^{-1 / 2}\left(u_{h}(t)-\tilde{u}_{h}(t)\right)\right\|_{0} \leq \frac{C}{t^{1 / 2}} H^{5}, \quad r \geq 3, \quad t \in(0, T] .
$$

Proof Let $y_{h}(t)=t^{1 / 2} A_{h}^{-1 / 2} e_{h}(t)$ and follow the arguments in the proof of Lemma 9 so that instead of (47) we now have

$$
\begin{aligned}
\left\|y_{h}(t)\right\|_{0} \leq & \int_{0}^{t} \frac{C}{\sqrt{t-s}} \| A_{h}^{-1} s^{1 / 2}\left(B_{h}\left(e_{h}, u_{H}\right)+B_{h}\left(u_{H}, e_{h}\right) \|_{0} d s\right. \\
& +\left\|\int_{0}^{t} e^{-(t-s) A_{h}} A_{h}^{-1 / 2} \Pi_{h} s^{1 / 2} \rho_{h, H}(s) d s\right\|_{0}, \\
& +\left\|\frac{1}{2} \int_{0}^{t} e^{-(t-s) A_{h}} s^{-1 / 2} A_{h}^{-1 / 2} e_{h}(s) d s\right\|_{0} .
\end{aligned}
$$

Now observe that by using $\left\|u_{H}(s)-u(s)\right\|_{j} \leq C H^{3-j} / s^{1 / 2}$, instead of (48) we now have

$$
\| A_{h}^{-1} s^{1 / 2}\left(B_{h}\left(e_{h}, u_{H}\right)+B_{h}\left(u_{H}, e_{h}\right) \|_{0} \leq C\left(\left\|y_{h}(s)\right\|_{0}+H^{3}\left\|e_{h}\right\|_{1}+H^{2}\left\|e_{h}\right\|_{0}\right)\right. \text {. }
$$

Thus, instead of (50) we now get

$$
\begin{aligned}
\max _{0 \leq t \leq T}\left\|y_{h}(t)\right\|_{0} \leq & C B\left(\frac{1}{2}, \frac{1}{2}\right)\left(\max _{0 \leq s \leq T} s\left\|A_{h}^{-1} \Pi_{h} \rho_{h, H}\right\|_{0}+\max _{0 \leq s \leq T}\left\|A_{h}^{-1} e_{h}(s)\right\|_{0}\right) \\
& +C\left(H^{3} \max _{0 \leq t \leq T}\left\|e_{h}\right\|_{1}+H^{2} \max _{0 \leq t \leq T}\left\|e_{h}\right\|_{0}\right) .
\end{aligned}
$$

Due to Lemma 15 we have that the last two terms on the right-hand side of (75) are $o\left(H^{5}\right)$, and due to Lemma 16 the second one is $O\left(H^{5}\right)$. Finally due to (68) the first one can be bounded by $C\left\|s^{1 / 2} \epsilon_{h, H}\right\|_{0}\left\|s^{1 / 2} \epsilon_{h, H}\right\|_{1}$, which, due to (25) is also $O\left(H^{5}\right)$.

Theorem 8 Let $(u, p)$ be the solution of (11)-(2). Then there exists a positive constant $C$ such that the approximations $u_{h}$ and $\tilde{u}_{h}$ to the velocity $u$ over the fine mesh satisfy the following bound:

$$
\left\|u_{h}(t)-\tilde{u}_{h}(t)\right\|_{0} \leq \frac{C}{t} H^{5}, \quad t \in(0, T], \quad r \geq 3 .
$$


Proof Let $y_{h}(t)=t e_{h}(t)$ and argue as in the proof of Theorem 1 so that similarly to (53) we now get

$$
\begin{aligned}
\max _{0 \leq t \leq T}\left\|y_{h}(t)\right\|_{0} \leq & C\left(\max _{0 \leq t \leq T}\left\|\int_{0}^{t} e^{-A_{h}(t-s)} s \Pi_{h} \rho_{h, H} d s\right\|_{0}\right. \\
& \left.+\max _{0 \leq t \leq T}\left\|\int_{0}^{t} e^{-A_{h}(t-s)} e_{h} d s\right\|_{0}\right) .
\end{aligned}
$$

Using (69) to bound the first term on the right-hand side above, and (16) for the second one, we get

$$
\begin{aligned}
\max _{0 \leq t \leq T}\left\|y_{h}(t)\right\|_{0} \leq & C\left(T^{1 / 4} \max _{0 \leq t \leq T}\left\|t^{1 / 2} \epsilon_{h, H}(t)\right\|_{0}\left\|t^{1 / 2} \epsilon_{h, H}(t)\right\|_{1}\right. \\
& \left.+B\left(\frac{1}{2}, \frac{1}{2}\right) \max _{0 \leq t \leq T}\left\|t^{1 / 2} A_{h}^{-1 / 2} e_{h}(t)\right\|_{0}\right),
\end{aligned}
$$

so that applying (25) and Lemma 17 the proof is finished.

Theorem 9 Let $(u, p)$ be the solution of (11)-(2). Then there exists a positive constant $C$ such that the approximations $u_{h}$ and $\tilde{u}_{h}$ to the velocity $u$ over the fine mesh satisfy the following bound:

$$
\left\|u_{h}(t)-\tilde{u}_{h}(t)\right\|_{1} \leq \frac{C}{t^{(r-1) / 2}} H^{r+1}, \quad t \in(0, T], \quad r=3,4 .
$$

Proof Let $y_{h}(t)=t^{(r-1) / 2} A_{h}^{1 / 2} e_{h}(t)$ and follow the arguments in the proof of Lemma 8 so that now, instead of (45) we get

$$
\begin{aligned}
\max _{0 \leq t \leq T}\left\|y_{h}(t)\right\|_{0} \leq & C\left(\max _{0 \leq t \leq T}\left\|\int_{0}^{t} e^{-(t-s) A_{h}} s^{(r-1) / 2} A_{h}^{1 / 2} \Pi_{h} \rho_{h, H}(s) d s\right\|_{0}\right. \\
& \left.+\frac{(r-1)}{2}\left\|\int_{0}^{t} e^{-(t-s) A_{h}} s^{(r-3) / 2} A_{h}^{1 / 2} e_{h}(s) d s\right\|_{0}\right) .
\end{aligned}
$$

Applying (70) to bound the first term on the right-hand side above and (16) for the second one, we have

$$
\begin{aligned}
\max _{0 \leq t \leq T}\left\|y_{h}(t)\right\|_{0} \leq & C\left(T^{1 / 4} \max _{0 \leq t \leq T}\left\|t^{1 / 2} \epsilon_{h, H}(t)\right\|_{1}\left\|t^{(r-2) / 2} \epsilon_{h, H}(t)\right\|_{1}\right. \\
& \left.+C B\left(\frac{1}{2}, \frac{1}{2}\right) \max _{0 \leq t \leq T}\left\|t^{(r-2) / 2} e_{h}(t)\right\|_{0}\right) .
\end{aligned}
$$

Due to (25) the first term on the right-hand side above is bounded by $C H^{2} H^{r-1}=C H^{r+1}$. For $r=4$, the second one is bounded in Theorem 8 , 
When $r=3$, we may write $\left\|t^{1 / 2} e_{h}(t)\right\|_{0}=\left\|t e_{h}(t)\right\|_{0}^{1 / 2}\left\|e_{h}(t)\right\|_{0}^{1 / 2}$ so that applying Theorem 8 and Lemma 15 , the second term on the right-hand side of (79) is bounded by $C H^{5 / 2} H^{7 / 4}=o\left(H^{4}\right)$

Remark 2 For $r=3$ it is possible to prove the bound

$$
\left\|u_{h}(t)-\tilde{u}_{h}(t)\right\|_{1} \leq \frac{C}{t}\left(H^{9 / 2}|\log (h)|+H^{17 / 4}\right), \quad t \in(0, T], \quad r=3 .
$$

To do so, apply Lemma 2 and (67) to bound the first term on the right-hand side of (78) and the same bound as before for the second term.

Finally, repeating (with obvious changes) the analysis in Section 3 for the pressure, the following result is easily proved

Theorem 10 Let $(u, p)$ be the solution of (1)-(2). There exists a positive constant $C$ such that the approximation to $p$ over the finer grid, $\tilde{p}_{h}$, satisfy the following bound for $t \in(0, T]$ and $r=3,4$ :

$$
\left\|\tilde{p}_{h}(t)-p_{h}(t)\right\|_{L^{2} / \mathbb{R}} \leq \frac{C}{t^{(r-1) / 2}} H^{r+1}, \quad t \in(0, T] .
$$

We now summarize the main results of the section in the following theorem.

Theorem 11 Let $(u, p)$ be the solution of (1)-(2). There exists a positive constant $C$ such that the approximations $\left(\tilde{u}_{h}, \tilde{p}_{h}\right)$ satisfy the following bounds for $r=3,4$ and $t \in(0, T]$ :

$$
\begin{aligned}
&\left\|u(t)-\tilde{u}_{h}(t)\right\|_{0} \leq \frac{C}{t} H^{5}+\frac{C}{t^{(r-2) / 2}} h^{r} . \\
&\left\|u(t)-\tilde{u}_{h}(t)\right\|_{1} \leq \frac{C}{t^{(r-1) / 2}} H^{r+1}+\frac{C}{t^{(r-2) / 2}} h^{r-1}, \\
&\left\|\tilde{p}_{h}(t)-p(t)\right\|_{L^{2} / \mathbb{R}} \leq \frac{C}{t^{(r-1) / 2}} H^{r+1}+\frac{C}{t^{(r-2) / 2}} h^{r-1} .
\end{aligned}
$$

Proof We use the decomposition $u-\tilde{u}=\left(u-u_{h}\right)+\left(u_{h}-\tilde{u}\right)$ and apply (25) to bound the first term and Theorems 8 and 9 for the second. For the pressure, using the decomposition $p-\tilde{p}_{h}=\left(p-p_{h}\right)+\left(p_{h}-\tilde{p}_{h}\right)$ and applying (60) and Theorem 10 the proof is finished.

Finally, with a much simpler analysis, that we do not detail here for brevity, the following result can be proved 
Theorem 12 Let $(u, p)$ be the solution of (1)-(2). There exists a positive constant $C$ such that the approximations over the finer grid computed using the mini-element, $\left(\tilde{u}_{h}, \tilde{p}_{h}\right)$, satisfy the following bounds for $t \in(0, T]$ :

$$
\begin{aligned}
\left\|\tilde{u}_{h}(t)-u(t)\right\|_{0} & \leq C H^{3}+C h^{2}, \\
\left\|\tilde{u}_{h}(t)-u(t)\right\|_{1} & \leq C H^{2}+C h \\
\left\|\tilde{p}_{h}(t)-p(t)\right\|_{L^{2} / \mathbb{R}} & \leq C H^{2}+C h .
\end{aligned}
$$

\section{Fully discrete case.}

In this section we consider the fully discrete case. Let us assume that we integrate in time equations $(17,-18)$ and $(19,-20)$ for the first method or equations (17, 18) and (62,63) for the second method using the backward Euler method or the two-step backward differentiation formula (BDF). In the case of the two-step BDF method the first step is carried out using the backward Euler method. We will denote by $\left(U_{H}^{n}, P_{H}^{n}\right)$ the fully discrete Galerkin approximations to the velocity and pressure at the time level $t_{n}=n k$ for $0 \leq n \leq N$ and $k=\Delta t=T / N$. We will denote by $\left(\widetilde{U}_{h}^{n}, \widetilde{P}_{h}^{n}\right)$ the fully discrete approximations to the velocity and pressure over the finer grid at the time level $t_{n}$.

Let us denote by $e_{H}^{n}=u_{H}\left(t_{n}\right)-U_{H}^{n}$ and by $\tilde{e}_{h}^{n}=\tilde{u}_{h}\left(t_{n}\right)-\widetilde{U}_{h}^{n}$ the temporal errors in the approximations $U_{H}^{n}$ and $\widetilde{U}_{h}^{n}$ respectively. Let us denote by $\pi_{H}^{n}=p_{H}\left(t_{n}\right)-P_{H}^{n}$ and by $\tilde{\pi}_{h}^{n}=\tilde{p}_{h}\left(t_{n}\right)-\widetilde{P}_{h}^{n}$ the temporal errors in the approximations $P_{H}^{n}$ and $\widetilde{P}_{H}^{n}$ respectively. In [15] we have proved the following error bounds. There exist constants $C_{l_{0}}$ and $k_{0}$ such that for $k \leq k_{0}$ the temporal errors of the Galerkin approximation satisfy the following error bounds

$$
\left\|e_{H}^{n}\right\|_{0}+t_{n}\left\|A_{H} e_{H}^{n}\right\|_{0} \leq C_{l_{0}} \frac{k^{l_{0}}}{t_{n}^{l_{0}-1}},\left\|\pi_{H}^{n}\right\|_{L^{2}(\Omega) / \mathbb{R}} \leq C_{l_{0}} \frac{k^{l_{0}}}{t_{n}^{\left(2 l_{0}-1\right) / 2}}, 1 \leq n \leq N,
$$

where $l_{0}=1$ for the Euler method and $l_{0}=2$ for the two-step BDF. Let us remark that using $\left\|e_{H}^{n}\right\|_{1} \leq C\left\|A_{H}^{1 / 2} e_{H}^{n}\right\|_{0} \leq C\left\|e_{H}^{n}\right\|_{0}^{1 / 2}\left\|A_{H} e_{H}^{n}\right\|_{0}^{1 / 2}$ error bounds in the $H^{1}$ norm are also obtained in a straightforward manner.

Using the same technique developed in [15] it can also be proved that analogous error bounds hold for the approximations over the finer grid. More precisely, for both the first and second algorithms there exist constants $C_{l_{0}}$ and $k_{0}$ such that for $k \leq k_{0}$ the temporal errors of the two-grid approxima- 
tion satisfy the following error bounds

$\left\|\tilde{e}_{h}^{n}\right\|_{0}+t_{n}\left\|A_{h} \tilde{e}_{h}^{n}\right\|_{0} \leq C_{l_{0}} \frac{k^{l_{0}}}{t_{n}^{l_{0}-1}},\left\|\tilde{\pi}_{h}^{n}\right\|_{L^{2}(\Omega) / \mathbb{R}} \leq C_{l_{0}} \frac{k^{l_{0}}}{t_{n}^{\left(2 l_{0}-1\right) / 2}}, 1 \leq n \leq N$,

where $l_{0}=1$ for the Euler method and $l_{0}=2$ for the two-step BDF.

Finally, using the decompositions

$$
\begin{aligned}
u\left(t_{n}\right)-\widetilde{U}_{h}^{n} & =\left(u\left(t_{n}\right)-\tilde{u}_{h}\left(t_{n}\right)\right)+\tilde{e}_{h}^{n}, \\
p\left(t_{n}\right)-\widetilde{P}_{h}^{n} & =\left(p\left(t_{n}\right)-\tilde{p}_{h}\left(t_{n}\right)\right)+\tilde{\pi}_{h}^{n},
\end{aligned}
$$

the error bounds of the fully discrete approximations are obtained as the sum of the spatial errors (the errors in the semi-discrete approximations we have already bounded in the previous sections) plus the temporal errors. 


\section{Bibliography}

[1] H. Abboud, V. Girault and T. Sayah, A second order accuracy for a full discretized time-dependet Navier-Stokes equations by a two-grid scheme, Numer. Math., 114 (2009), pp. 189-231.

[2] H. Abboud and T. Sayah, A full discretization of the time-dependent Navier-Stokes equations by a two-grid scheme, M2AN Math. Model. Numer. Anal., 42 (2008), pp. 141-174.

[3] P. J. DAvis, Gamma function and related functions, in Handbook of Mathematical Functions with Formulas, Graphs, and Mathematical Tables, M. Abramowitz and I. A. Stegun, eds., Dover, New York, 1992 (reprint of the 1972 edition).

[4] R. Adams, Sobolev Spaces, Academic Press, New York, 1975.

[5] A. Ait Ou Amni And M. Marion, Nonlinear Galerkin methods and mixed finite element: two-grid algorithms for the Navier-Stokes equations, Numer. Math., 62 (1994), pp. 189-213.

[6] B. Ayuso, J. De Frutos, And J. Novo, Improving the accuracy of the mini-element approximation to Navier-Stokes equations, IMA J. Numer. Anal., 27 (2007), pp. 198-218.

[7] B. Ayuso, B. García-Archilla, and J. Novo, The postprocessed mixed finite-element method for the Navier-Stokes equations, SIAM J. Numer. Anal., 43 (2005), pp. 1091-1111.

[8] F. Brezzi And R. S. FALK, Stability of higher-order Hood-Taylor methods, SIAM J. Numer. Anal., 28 (1991), pp. 581-590.

[9] F. Brezzi And M. Fortin, Mixed and Hybrid Finite Element Methods, Springer, New York, 1991. 
[10] P. G. Ciarlet, The Finite Element Method for Elliptic Problems, North-Holland, Amsterdam, 1978.

[11] P. Constantin and C. Foias, Navier-Stokes Equations, Chicago Lectures in Math., The University of Chicago, Chicago, 1988.

[12] C. Devulder, M. Marion and E. S. Titi, On the rate of convergence of the Nonlinear Galerkin methods, Math. Comput., 60 (1993), pp. 495-514.

[13] J. De Frutos, B. García-Archilla and J. Novo, A postprocessed Galerkin method with Chebyshev or Legendre polynomials, Numer. Math., 86 (2000), pp. 419-442.

[14] J. De Frutos, B. García-Archilla and J. Novo, The postprocessed mixed finite-element method for the Navier-Stokes equations: refined error bounds, SIAM J. Numer. Anal., 46 (2007), pp. 201-230.

[15] J. de Frutos, B. García-Archilla and J. Novo, Postprocessing finite-element methods for the Navier-Stokes equations: the fully discrete case, SIAM J. Numer. Anal., 47 (2008), pp. 596-621.

[16] J. De Frutos And J. Novo, A spectral element method for the NavierStokes equations with improved accuracy, SIAM J. Numer. Anal., 38 (2000), pp. 799-819.

[17] H. Fujita And T. Kato, On the Navier-Stokes initial value problem. I, Arch. Ration. Mech. Anal., 16 (1964), pp. 269-315.

[18] B. García-Archilla, J. Novo, And E. S. Titi, Postprocessing the Galerkin method: A novel approach to approximate inertial manifolds, SIAM J. Numer. Anal., 35 (1998), pp. 941-972.

[19] B. García-Archilla, J. Novo, and E. S. Titi, An approximate inertial manifold approach to postprocessing Galerkin methods for the Navier-Stokes equations, Math. Comp., 68 (1999), pp. 893-911.

[20] V. Girault and J. L. Lions, Two-grid finite-element schemes for the transient Navier-Stokes problem, M2AN Math. Model. Numer. Anal., 35 (2001), pp. 945-980.

[21] Y. HE, Two-level method based on finite element and Crank-Nicolson extrapolation for the time-dependent Navier-Stokes equations, SIAM J. Numer. Anal., 41 (2003), pp. 1263-1285. 
[22] D. Henry, Geometric Theory of Semilinear Parabolic Equations, Springer-Verlag, Berlin, 1991.

[23] J. G. Heywood and R. Rannacher, Finite element approximation of the nonstationary Navier-Stokes problem. I. Regularity of solutions and second-order error estimates for spatial discretization, SIAM J. Numer. Anal., 19 (1982), pp. 275-311.

[24] J. G. Heywood And R. Rannacher, Finite element approximation of the nonstationary Navier-Stokes problem. III: Smoothing property and higher order error estimates for spatial discretization, SIAM J. Numer. Anal., 25 (1988), pp. 489-512.

[25] J. G. Heywood And R. RAnnaCher, Finite-element approximation of the nonstationary Navier-Stokes problem. IV: Error analysis for second-order time discretization, SIAM J. Numer. Anal., 27 (1990), pp. 353-384.

[26] P. Hood And C. TAYlor, A numerical solution of the Navier-Stokes equations using the finite element technique, Comput. Fluids, 1 (1973), pp. $73-100$.

[27] Y. Hou And K. Li, Postprocessing Fourier Galerkim method for the Navier-Stokes equations, SIAM. J. Numer. Anal., 47 (2009), pp. 19091922.

[28] W. Layton and L. Tobiska, A Two-Level method with backtracking for the Navier-Stokes equations, SIAM J. Numer. Anal., 35 (1998), pp. 2035-2054.

[29] W. Layton and W. Lenferink, Two-Level Picard and Modified Picard Methods for the Navier-Stokes Equations, Appl. Math. Comput., 80 (1995), pp. 1-12.

[30] Q. Liu And Y. Hou, A two-level finite element method for the NavierStokes equations based on a new projection, Applied Mathematical Modelling, 34 (2010) pp. 383-399.

[31] L. G. Margolin, E. S. Titi and S. Wynne, The postprocessing Galerkin and nonlinear Galerkin methods-A truncation analysis point of view, SIAM J. Numer. Anal., 41 (2003), pp. 695-714.

[32] M. Marion and R. Temam, Nonlinear Galerkin Methods, SIAM J. Numer. Anal., 26, 1989, pp. 1139-1157. 
[33] M. A. Olshanskit, Two-level method and some a priori estimates in unsteady Navier-Stokes calculations, J. Comp. Appl. Math., 104 (1999), pp. 173-191.

[34] J. XU, A novel two-grid method for semilinear elliptic equations, SIAM J. Sci. Comput., 15 (1994), pp 231-237.

[35] J. XU, Two grid discretizations for linear and nonlinear PDE., SIAM J. Numer. Anal., 33 (1996), pp. 1759-1777. 